\title{
Rapamycin improves TIE2-mutated venous malformation in murine model and human subjects
}

\author{
Elisa Boscolo, ${ }^{1}$ Nisha Limaye, ${ }^{2}$ Lan Huang, ${ }^{1}$ Kyu-Tae Kang, ${ }^{1}$ Julie Soblet, ${ }^{2}$ Melanie Uebelhoer, ${ }^{2}$ Antonella Mendola, ${ }^{2}$ Marjut Natynki, \\ Emmanuel Seront, ${ }^{4}$ Sophie Dupont, ${ }^{4}$ Jennifer Hammer, ${ }^{5}$ Catherine Legrand, ${ }^{6}$ Carlo Brugnara, ${ }^{7}$ Lauri Eklund, ${ }^{3}$ Miikka Vikkula, ${ }^{2,5}$ \\ Joyce Bischoff, ${ }^{1}$ and Laurence M. Boon ${ }^{2,5}$ \\ 'Vascular Biology Program and Department of Surgery, Boston Children's Hospital, Harvard Medical School, Boston, Massachusetts, USA. ${ }^{2 H u m a n ~ M o l e c u l a r ~ G e n e t i c s, ~ d e ~ D u v e ~ I n s t i t u t e, ~}$ \\ Université catholique de Louvain, Brussels, Belgium. ${ }^{3}$ Oulu Center for Cell-Matrix Research, Biocenter Oulu and Department of Medical Biochemistry and Molecular Biology, University of Oulu, \\ Oulu, Finland. ${ }^{4}$ Centre du Cancer, Department of Pediatric Oncology, and ${ }^{5}$ Center for Vascular Anomalies, Division of Plastic Surgery, Cliniques Universitaires Saint Luc, Université catholique de Louvain, \\ Brussels, Belgium. ${ }^{6}$ Institute of Statistics, Biostatistics, and Actuarial Sciences, Université catholique de Louvain, Louvain-la-Neuve, Belgium. 'Department of Laboratory Medicine, \\ Boston Children's Hospital, Harvard Medical School, Boston, Massachusetts, USA.
}

Venous malformations (VMs) are composed of ectatic veins with scarce smooth muscle cell coverage. Activating mutations in the endothelial cell tyrosine kinase receptor TIE2 are a common cause of these lesions. VMs cause deformity, pain, and local intravascular coagulopathy, and they expand with time. Targeted pharmacological therapies are not available for this condition. Here, we generated a model of VMs by injecting HUVECs expressing the most frequent VM-causing TIE2 mutation, TIE2-L914F, into immune-deficient mice. TIE2-L914F-expressing HUVECs formed VMs with ectatic blood-filled channels that enlarged over time. We tested both rapamycin and a TIE2 tyrosine kinase inhibitor (TIE2-TKI) for their effects on murine VM expansion and for their ability to inhibit mutant TIE2 signaling. Rapamycin prevented VM growth, while TIE2-TKI had no effect. In cultured TIE2-L914F-expressing HUVECs, rapamycin effectively reduced mutant TIE2-induced AKT signaling and, though TIE2-TKI did target the WT receptor, it only weakly suppressed mutant-induced AKT signaling. In a prospective clinical pilot study, we analyzed the effects of rapamycin in 6 patients with difficult-to-treat venous anomalies. Rapamycin reduced pain, bleeding, lesion size, functional and esthetic impairment, and intravascular coagulopathy. This study provides a VM model that allows evaluation of potential therapeutic strategies and demonstrates that rapamycin provides clinical improvement in patients with venous malformation.

\section{Introduction}

Venous malformations (VMs) are composed of ectatic and distorted veins surrounded by sparse and irregularly distributed smooth muscle cells $(1,2)$. VMs form compressible bluish lesions that are usually present at birth and continue to grow with the child. Rarely, patients have multifocal lesions that increase in number with time. Many VMs cause chronic painful swelling, obstruction of vital structures, and bleeding $(3,4)$. Localized intravascular coagulopathy (LIC) occurs in one-half of patients with large and/ or multifocal VMs; D-dimer level is increased and, in severe cases, fibrinogen level is low (4-7). Pulmonary embolism can occur in VMs with large draining veins (8). Diagnosis of VM is performed through clinical evaluation, Doppler ultrasonography, and MRI. Among vascular anomalies, LIC is specific to VMs and elevated $\mathrm{D}$-dimer level is a useful biomarker for differential diagnosis and evaluation of treatment efficacy (4-6). Sclerotherapy is the primary treatment, alone or in combination with surgical resection $(3,4)$. Sclerotherapy can cause swelling, skin necrosis, peripheral

Authorship note: Miikka Vikkula, Joyce Bischoff, and Laurence M. Boon contributed equally to this work.

Conflict of interest: The authors have declared that no conflict of interest exists. Submitted: May 26, 2015; Accepted: July 2, 2015.

Reference information: J Clin Invest. 2015;125(9):3491-3504. doi:10.1172/JCI76004. nerve deficits, and rarely, cardiac arrest, depending on the sclerosing agent used (9-12). Extensive VMs commonly persist after treatment (4). Since current treatments are rarely curative, the aim is to alleviate signs and symptoms.

Familial mucocutaneous venous malformation (VMCM) (13) and $50 \%$ of sporadic VMs are caused by mutations in the tyrosine kinase (TK) receptor TIE2 (13-16). The most common inherited VMCM mutation is R849W $(13,14,16,17)$. Sporadic VMs are more frequent ( $>98 \%$ of patients) and typically consist of extensive unifocal or, rarely, multifocal lesions. VM-causative mutations are located in the TK domain, kinase-insert (KID), and carboxy terminal (C-terminal) tail domains and cause ligand-independent activation of TIE2 (13-17). The somatic mutation L914F accounts for $77 \%$ of patients with mutation-positive lesions $(14,15)$.

The TIE/angiopoietin (ANGPT) family includes 2 receptors (TIE1 and TIE2) and 3 ligands (ANGPT1, ANGPT2, and ANGPT4) $(18,19)$. TIE2 is expressed in endothelial cells, hematopoietic stem cells (20), and proangiogenic monocytes (21). ANGPT1 and ANGPT2 bind to TIE2 and mediate, respectively, vascular maturation and angiogenesis (22-25). Knockout of Tie2 or Angpt-1 in mice results in impaired blood vessel branching and deficient perivascular coverage $(25,26)$. Deletion of Angpt-1 in the developing embryo produces a disorganized vascular network with an increased number of ectatic vessels (27). 

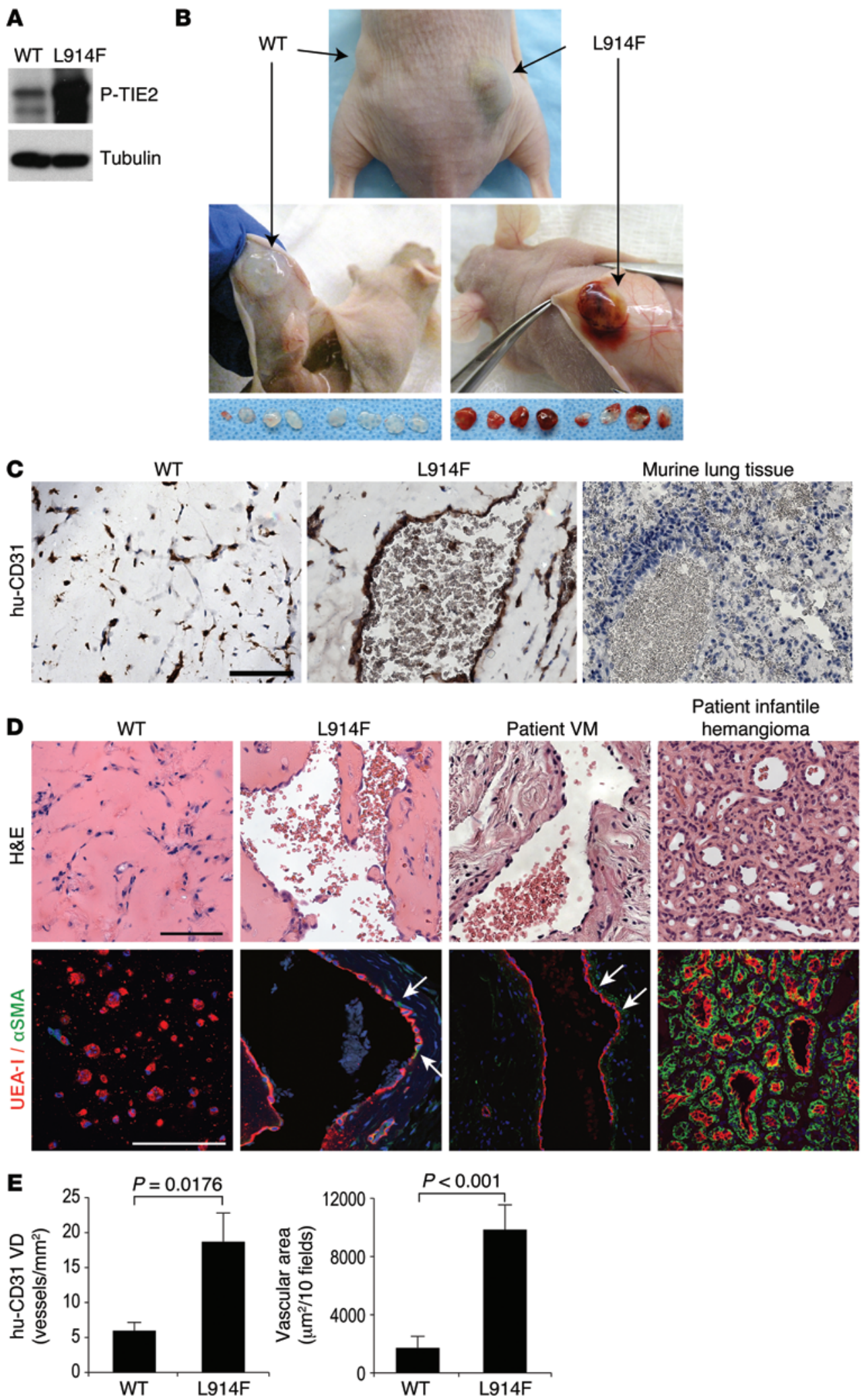

Figure 1. HUVEC-TIE2-L914F formed

VM lesions in immune-deficient mice.

(A) Western blot of $\mathrm{p}$-TIE2 in HUVECs transfected with TIE2-WT or with mutant TIE2 (L914F). Tubulin served as loading control. (B) Representative nude mouse 7 days after injection of HUVEC-TIE2-WT (left flank) or HUVECTIE2-L914F (right flank) and Matrigel explants from each injected mouse $(n=8)$ (bottom panel). (C) Matrigel explants with HUVEC-TIE2-WT (left) or HUVEC-TIE2-L914F (middle) sectioned and stained with specific anti-human CD31 (hu-CD31), representative images ( $n=4)$. Murine lung tissue (right) is shown as negative control for antihuman CD31 staining. Scale bar: 100 $\mu \mathrm{m}$. (D) HUVEC-TIE2-WT and HUVECTIE2-L914F tissue sections stained for $\mathrm{H} \& \mathrm{E}$ (top panel) and UEA-I and aSMA (bottom panel). Patient-derived VM and infantile hemangioma are shown for comparison. Representative images ( $n=4$ WT, L914F; $n=2$ patients with $\mathrm{VM} ; n=3$ patients with infantile hemangioma). UEA-I (red) and $\alpha S M A$ (green); arrows point to areas with perivascular $\alpha \mathrm{SMA}^{+}$cell coverage in $\mathrm{VM}$ vessels. Scale bars: $100 \mu \mathrm{m}$. (E) Quantification of human CD31-stained blood vessels (vessels $/ \mathrm{mm}^{2}$ ) and luminal area occupied by human CD31-stained blood vessels $\left(\mu \mathrm{m}^{2}\right)$ in HUVEC-TIE2-WT or HUVEC-TIE2-L914F mid-explant sections. Data expressed as mean \pm SEM, $t$ test $(n=8)$.
Previous studies on mutant TIE2 showed that expression of TIE2-L914F or TIE2-R849W in HUVECs increased activation of AKT and of STAT-1 (28-32). Elevated AKT signaling in these cells has been linked to increased survival $(29,30)$ and to reduced production of PDGF-B (30), a major player in mural cell recruitment.
Other factors are likely involved, since over 80 genes were differentially expressed (at least 2-fold) in the TIE2-L914F endothelial cells compared with WT (30).

We hypothesized that mutant TIE2 in endothelial cells is sufficient to cause VM. Here, we show that HUVECs engineered to 

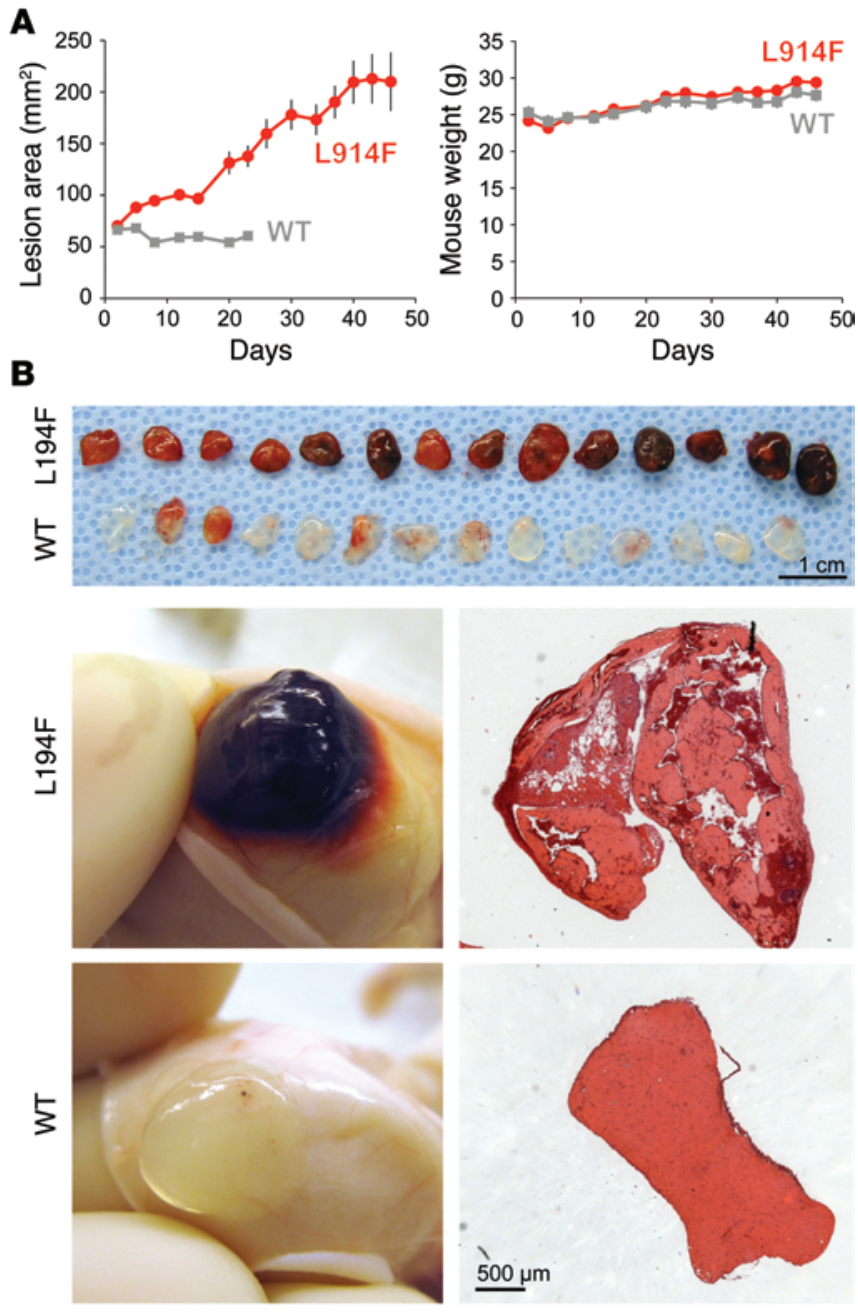

C
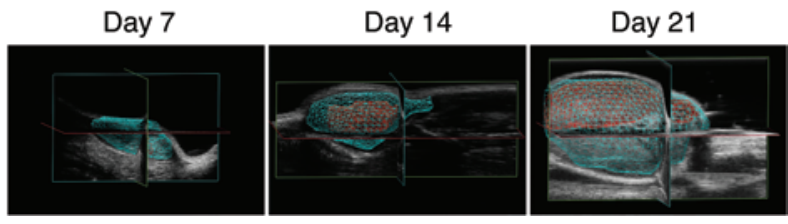

express TIE2-L914F recapitulate VM when injected in immunedeficient mice, while HUVECs induced to express WT TIE2 (TIE2-WT) do not. We tested the effects of rapamycin and a TIE2 tyrosine kinase inhibitor (TIE2-TKI) on murine VM formation and enlargement using 3 different treatment schemes. Rapamycin decreased both lesion growth and vascular volume in VMs, whereas TIE2-TKI had a mild effect in limiting VM vascular volume. In vitro, TIE2-TKI showed dose-dependent inhibition of TIE2 and AKT-Thr308/-Ser473 phosphorylation in HUVEC-TIE2-WT and HUVEC-TIE2-R1099X, but only a weak ability to inhibit the constitutive phosphorylation of AKT-Thr308/-Ser473 mediated by the mutant TIE2-L914F. In contrast, rapamycin effectively reduced AKT-Ser473 phosphorylation. In our prospective clinical pilot study, which included 6 patients with VMs that were refractory to standard care ( $n=2$ TIE2-L914F, $n=1$ TIE2-R1099X, $n=3$ no TIE2 mutation), rapamycin reduced pain, LIC, and bleeding, functional and esthetic impairment, and radiological lesion size, suggesting it could be the first molecular therapy for VMs.
Figure 2. HUVEC-TIE2-L914F assembled in enlarged channels filled with blood. (A) HUVEC-TIE2-L914F (red) and HUVEC-TIE2-WT (gray) lesional area measured by caliper every 3 to 5 days for 50 days. HUVEC-TIE2-WT lesions were undetectable after day 23. Mouse weight monitored for 50 days. Data expressed as mean \pm SEM, $t$ test $(n=10$ mice with 2 lesions each/group). (B) Lesions explanted 14 days after injection ( $n=7$ mice with 2 lesions/group). Top row: HUVEC-TIE2-L914F; bottom row: HUVEC-TIE2WT; below, left: representative images of lesions; below, right: H\&Estained sections from middle of the explant. Scale bars: $1 \mathrm{~cm}$ (top panel); $500 \mu \mathrm{m}$ (bottom panels). (C) Representative color Doppler 3D image stacks of murine VM lesion analyzed at days 7,14 , and 21 ( $n=3$ mice were analyzed with 3D color Doppler at days 7, 14, and 21).

\section{Results}

TIE2 mutation L914F is sufficient to induce HUVECs to form VM lesions in mice. We expressed mutated (L914F) and TIE2-WT receptor in HUVECs by retroviral gene transfer. HUVEC-TIE2-L914F showed constitutively increased TIE2 phosphorylation (Figure 1A), as previously reported (14). We injected HUVEC-TIE2-WT or HUVECTIE2-L914F, resuspended in Matrigel, s.c. in immune-deficient $\mathrm{nu} / \mathrm{nu}$ mice in the left and right flanks, respectively. At day 7, 8 of 8 explants with HUVEC-TIE2-L914F were vascularized, while 8 of 8 explants with HUVEC-TIE2-WT were not (Figure 1B). In the TIE2L914F lesions, the vascular channels were positive for the humanspecific anti-CD31 (Figure 1C). Human CD31 ${ }^{+}$cells were detected in HUVEC-TIE2-WT explants, but only rare vessels were observed. Blood vessels in HUVEC-TIE2-L914F explants were ectatic $(>50$ $\mu \mathrm{m}$ in diameter), similarly to those of patients with VM (Figure 1D). Furthermore, a thin, scarce, noncontinuous layer of $\alpha \mathrm{SMA}^{+}$perivascular cells surrounded large ulex europaeus agglutinin- $\mathrm{I}^{+}\left(\mathrm{UEA}-\mathrm{I}^{+}\right)$ channels in the HUVEC-TIE2-L914F lesions (Figure 1D). This phenotype closely resembled VM lesions in patients and was distinct from the highly vascularized tissue in infantile hemangioma (Figure 1D and Supplemental Figure 1; supplemental material available online with this article; doi:10.1172/JCI76004DS1). Moreover, inflammatory cells were not evident in either patient or murine VM tissue sections (Figure 1D and Supplemental Figure 2). Quantitative analysis revealed that human $\mathrm{CD} 31^{+}$vessel density (VD) and vascular area were significantly $(P<0.05)$ higher in HUVEC-TIE2-L914F explants compared with HUVEC-TIE2-WT (Figure 1E).

Murine VM lesions enlarge over time. HUVEC-TIE2-L914F lesions enlarged in mice over 50 days (Figure 2A). Conversely, HUVEC-TIE2-WT implants did not grow and became undetectable after day 23. Body weight was similar in both groups of mice. Images of lesional explants at day 14 (Figure 2B) illustrate that 14 of 14 HUVEC-TIE2-L914F lesions were vascularized and bigger than the HUVEC-TIE2-WT lesions; on histology, they contained massively enlarged blood-filled channels (Figure 2B). In contrast,

Table 1. Analysis of murine VM lesion size

$\begin{array}{llll} & \text { Day 7 } & \text { Day 14 } & \text { Day } 21 \\ \text { Total volume }\left(\mathrm{mm}^{3}\right) & 102.097 & 226.525 & 1339.871 \\ \text { Vascular volume }\left(\mathrm{mm}^{3}\right) & 0.993 & 51.349 & 235.228\end{array}$

Total volume and vascular volume of a representative murine VM lesion at days 7,14 , and 21. Analysis was performed through color Doppler 3D image stacks. 
A

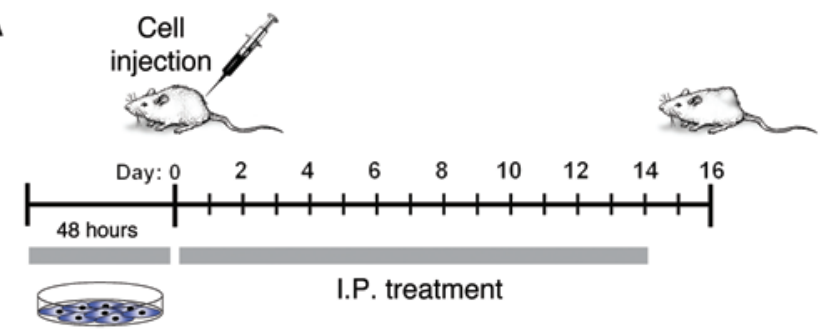

Cell pretreatment

B

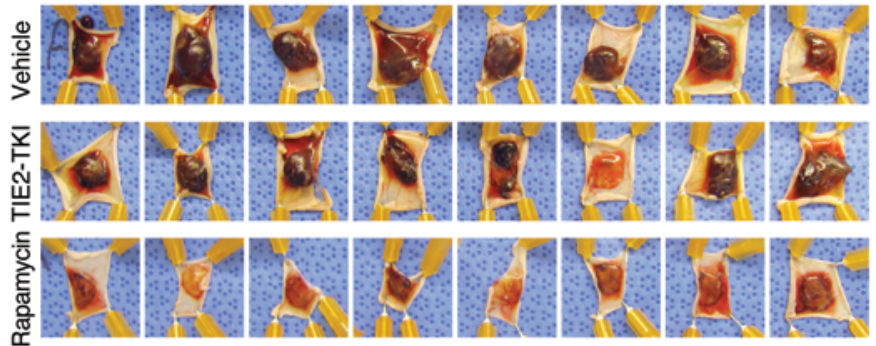

C
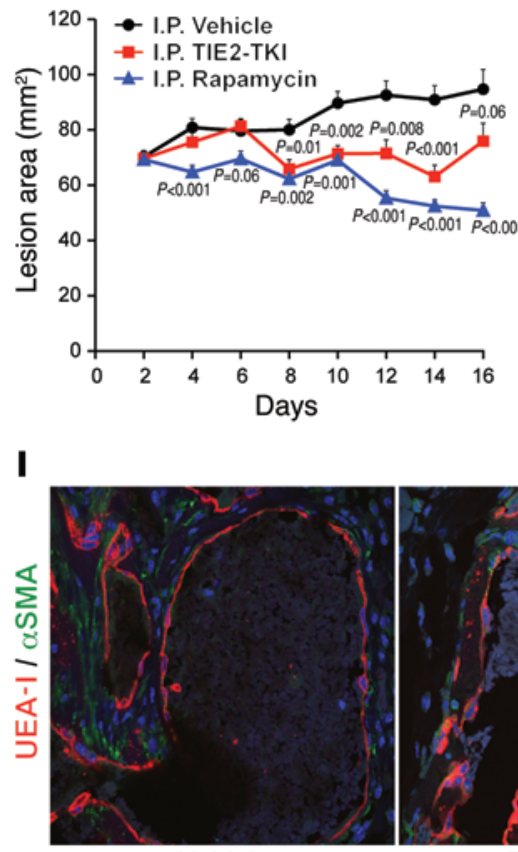

Vehicle
D

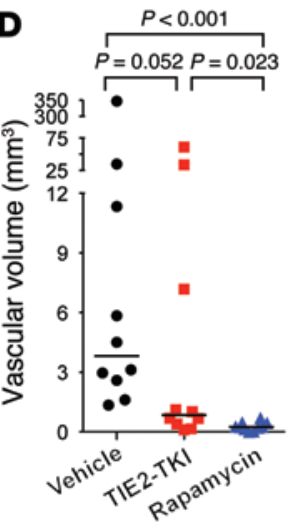

E

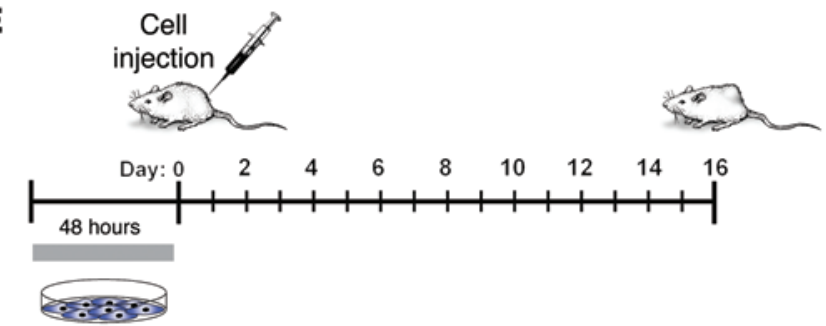

Cell pretreatment

F

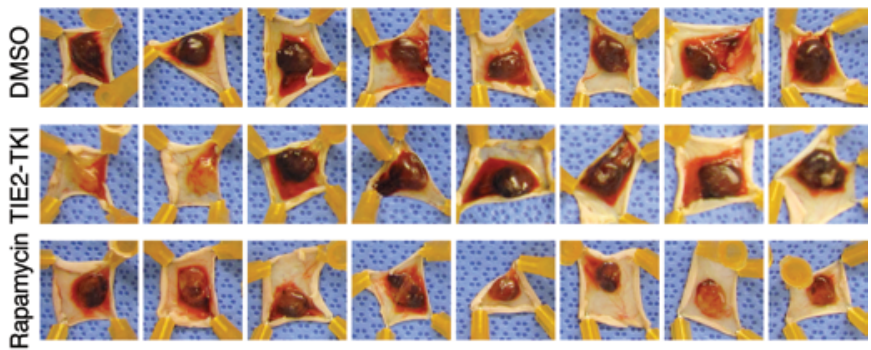

G

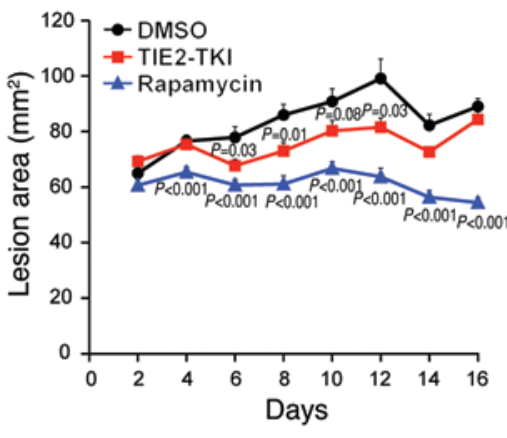

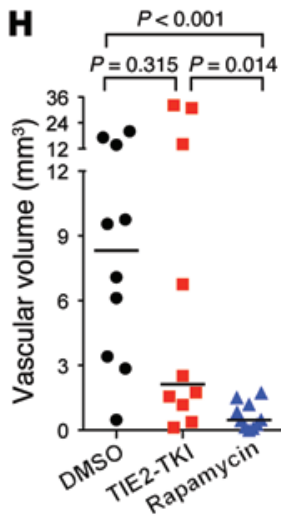

J

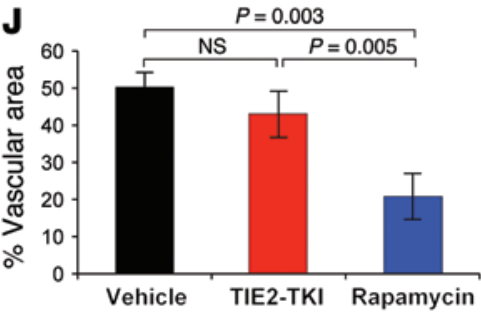

K

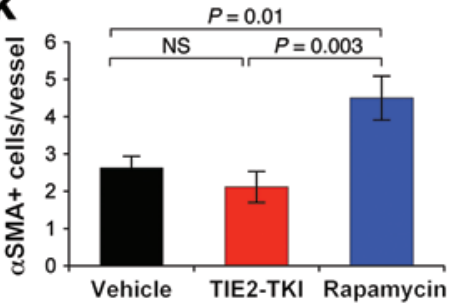

Figure 3. TIE2-TKI and rapamycin effects on murine VMs. (A) Pretreatment plus i.p. injection schematic. (B) Representative murine VM lesion explants at day 16. Top row: vehicle; middle row: TIE2-TKI; bottom row: rapamycin. Images taken from fixed distance. (C) HUVEC-TIE2-L914F lesional area measured by caliper every 2 days for 16 days. Data expressed as mean \pm SEM, $t$ test ( $n=5$ mice with 2 lesions/group). (D) Vascular volume at day 15 measured by analysis of color Doppler $3 D$ image stacks. Data expressed as single values for each lesion ( $n=5$ mice with 2 lesions/group); medians shown by horizontal bars, Mann-Whitney $U$ test. (E) Pretreatment schematic. (F) Representative murine VM lesion explants at day 16. Top row, DMSO; middle row, TIE2-TKI; bottom row, rapamycin. Images taken from fixed distance. (C) HUVEC-TIE2-L914F lesional area measured by caliper every 2 days for 16 days. Data expressed as mean $\pm S E M, t$ test ( $n=5$ mice with 2 lesions/group; shown is a representative experiment from 2 independent experiments). (H) Vascular volume at day 16 measured by analysis of color Doppler $3 D$ image stacks. Data expressed as single values for each lesion; medians shown by horizontal bars, Mann-Whitney $U$ test ( $n=5$ mice with 2 lesions/group; shown is a representative experiment from 2 independent experiments). (I) Representative images of VM sections immunostained for UEA-I (red), $\alpha$ SMA (green), and DAPI (blue). Arrows point to $\alpha \mathrm{SMA}^{+}$cells. Scale bar: $100 \mu \mathrm{M}$. (J) Quantification of luminal area occupied by UEA-I-stained blood vessels, expressed as percentage of total sectional area. Data expressed as mean \pm SEM, $t$ test ( $n=5$ fields/section, 7 mice analyzed/group). NS, $P>0.05$. (K) Quantification of $\alpha S^{2} A^{+}$cells surrounding UEA-I-stained blood vessels. Data expressed as mean \pm SEM ( $n=5$ fields/section, 7 mice analyzed/group). NS, $P>0.05$. 
A

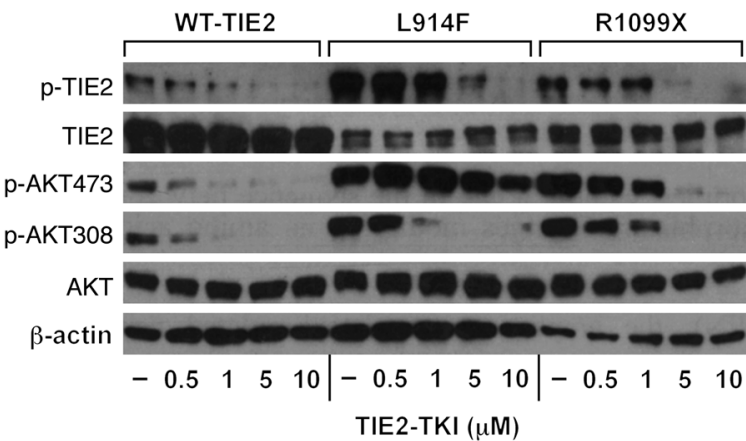

B
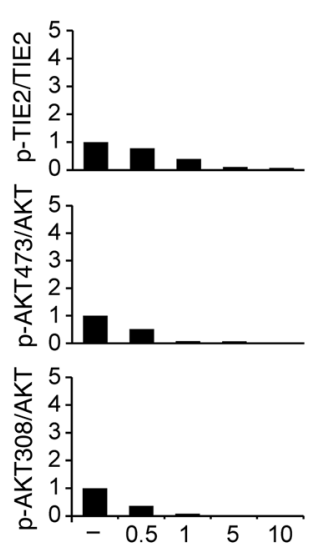

L914F
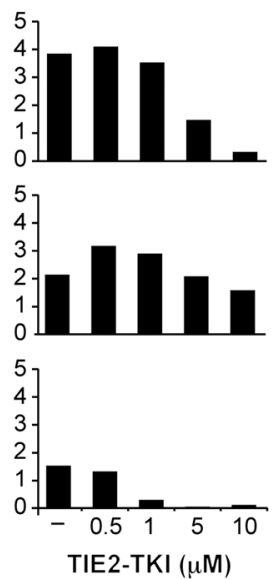

R1099X
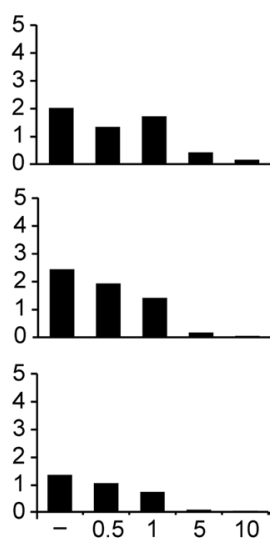

C

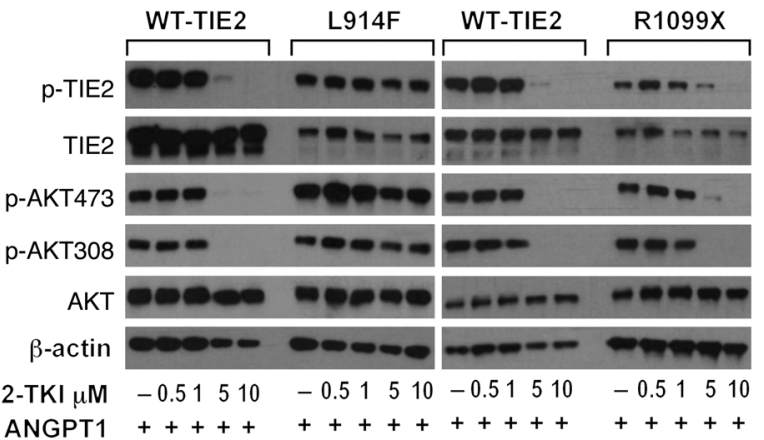

D
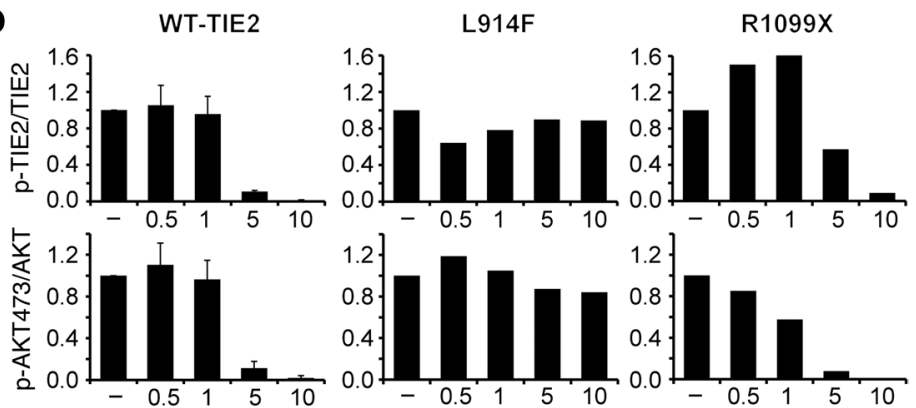
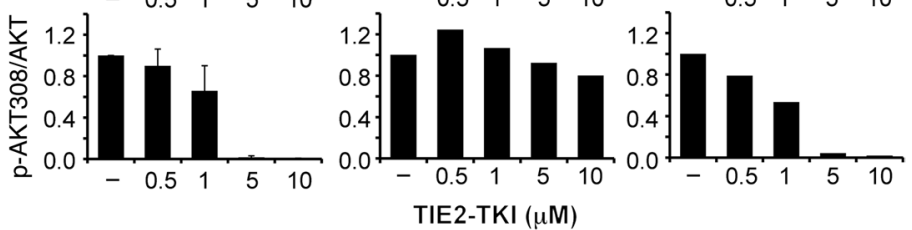

Figure 4. TIE2-TKI effects on TIE2 and AKT phosphorylation in TIE2-WT, TIE2-L914F, and TIE2-R1099X mutant HUVECs. (A) Western blot analysis of HUVECTIE2-WT, HUVEC-TIE2-L914F, and HUVEC-TIE2-R1099X after 48 hours treatment with TIE2-TKI (0.5-10 $\mu \mathrm{M})$. $\beta$-Actin served as loading control. (B) Densitometric analysis of p-TIE2, p-AKT473, and p-AKT308 Western blot bands relative to total TIE2 and AKT, respectively. Data are normalized to untreated HUVEC-TIE2WT. (C) Western blot analysis of HUVEC-TIE2-WT, HUVEC-TIE2-L914F, and HUVEC-TIE2-R1099X pretreated for 2 hours with TIE2-TKI (0.5-10 $\mu$ M), subsequently stimulated for 15 minutes with $1 \mu \mathrm{g} / \mathrm{ml}$ ANGPT1. $\beta$-Actin served as loading control. (D) Densitometric analysis of p-TIE2, p-AKT473, and p-AKT308 Western blot bands relative to total TIE2 and AKT, respectively. Data are normalized to untreated, $1 \mu \mathrm{g} / \mathrm{ml}$ ANGPT1 conditions.

HUVEC-TIE2-WT implants showed only a few areas of vascularization. Color Doppler 3D image stacks of a representative murine VM lesion were acquired at 7, 14, and 21 days (Figure 2C). Total volume and vascular volume increased from day 7 to days 14 and 21 (Table 1). Furthermore, Doppler ultrasonography analysis showed that VM blood flow was slow and nonpulsatile, akin to VM lesions in patients (Supplemental Videos 1 and 2). Blood drawn from murine lesions also showed characteristics of venous blood, as indicated by high $\mathrm{pCO}_{2}$ and low $\mathrm{pO}_{2}$ (Supplemental Table 1). In addition, peripheral blood from mice bearing VMs displayed a significantly $(P<0.05)$ higher number of reticulocytes $(1,090,000$ $\pm 210,000$ vs. $250,000 \pm 30,000$ cells/ $\mu$ l), lower erythrocyte counts $(6,460,000 \pm 450,000$ vs. $8,520,000 \pm 130,000$ cells/ $\mu l)$, and lower hemoglobin (Hb: $10.94 \pm 0.63 \mathrm{vs} .13 .2 \pm 0.15 \mathrm{~g} / \mathrm{dl}$ ) when compared with control mice (Supplemental Table 2).

Rapamycin reduces expansion of HUVEC-TIE2-L914F VM lesions. Rapamycin and TIE2-TKI were tested in the VM murine model. HUVEC-TIE2-L914F were pretreated in vitro for 48 hours with vehi- cle, TIE2-TKI, or rapamycin, and, after cell injection, mice were given vehicle, TIE2-TKI $(10 \mathrm{mg} / \mathrm{kg})$, or rapamycin $(2 \mathrm{mg} / \mathrm{kg})$ daily by i.p. injection from day 1 to day 14 (schematic in Figure 3A). We reached the maximum solubility of TIE2-TKI compound in vehicle at $10 \mathrm{mg} /$ $\mathrm{kg}$, a dose effective for reducing tumor growth of MOPC-315 xenografts (33). The rapamycin injected dose was $2 \mathrm{mg} / \mathrm{kg}$, which was found effective in preventing blood vessel formation in a murine model of infantile hemangioma (34) and in preventing tumor growth and vascular permeability in a model of glioma (35). At day 16, VM lesions from the rapamycin and TIE2-TKI-treated groups appeared smaller and less vascularized than the vehicle-treated lesions (Figure 3B). When compared with the vehicle-treated group, the lesional area was significantly smaller $(P<0.05)$ in the rapamycin-treated group from day 4 to day 16 and in the TIE2-TKI-treated group $(P<0.05)$ from day 8 to day 14 (Figure 3C). At day 16, the vascular volume of lesions in the rapamycin-treated group was significantly lower $(P<0.001)$ than in the vehicle- and the TIE2-TKI-treated $(P<0.05)$ groups (Figure 3D). Vehicle- and TIE2-TKI-treated mice 
A

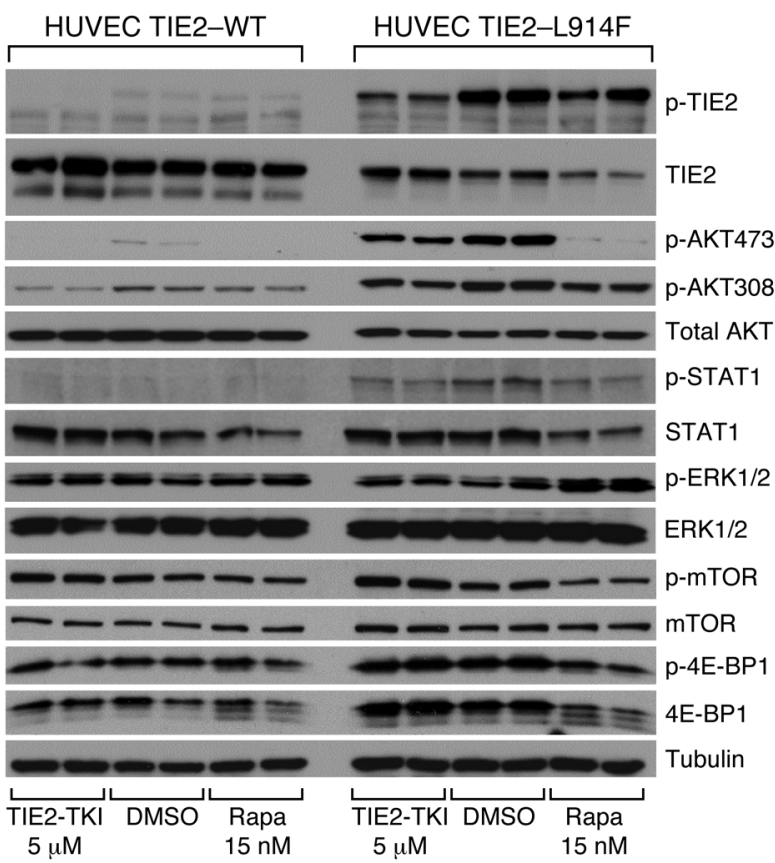

Figure 5. TIE2-TKI and rapamycin effects on signaling pathways downstream of TIE2 in HUVEC-TIE2-WT and HUVEC-TIE2-L914F. (A) Immunoblot analysis of HUVEC-TIE2-WT and TIE2-L914F treated for 48 hours with TIE2-TKI ( $5 \mu \mathrm{M})$, DMSO, or rapamycin ( $15 \mathrm{nM})$. Two protein samples loaded from 2 different cell treatment sets. Tubulin served as loading control. (B) Densitometric analysis of p-TIE2, p-AKT473, p-AKT308, p-STAT1, p-ERK, p-mTOR, and p-4E-BP1 Western blot bands relative to total protein, TIE2, AKT, STAT1, ERK, mTOR, and 4E-BP1, respectively. Data expressed as mean $\pm \mathrm{SD}, t$ test $(n=4,2$ independent experiments). Data are normalized to DMOS-treated HUVEC-TIE2-WT (dashed lines).
B
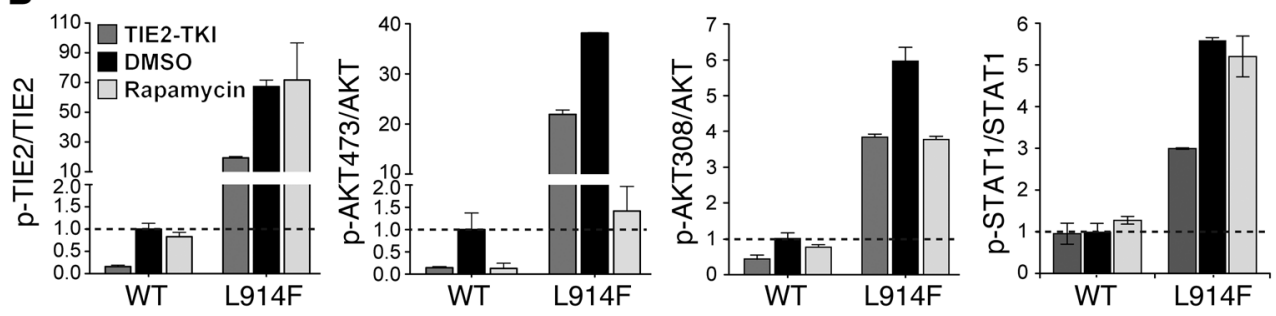

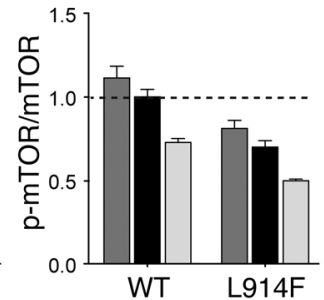

WT

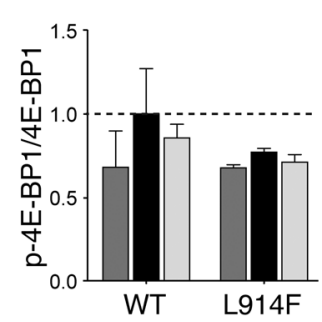

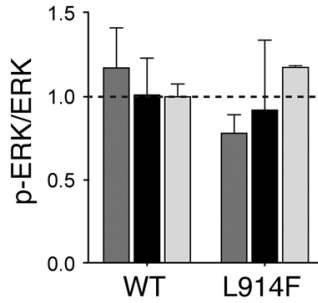

exhibited lesions with a highly variable vascular volume; rapamycin treatment, by preventing lesional growth, greatly reduced this variability. The TIE2-TKI-treated group showed a trend toward reduced vascular volume $(P=0.052)$ compared with the vehicle-treated group.

In order to study the specific effect of rapamycin and TIE2-TKI on the TIE2-L914F mutant endothelium independently of effects on murine cells, we repeated the experimental scheme shown in Figure 3A, but i.p. drug injections were omitted. In detail, HUVEC-TIE2L914F were pretreated in vitro for 48 hours with rapamycin, TIE2TKI, or DMSO, collected, and injected into mice (schematic in Figure 3E). At day 16, explants from all 3 groups showed vascularization, although it was seemingly less prominent in the rapamycin-treated group (Figure 3F). Lesional area was significantly lower $(P<0.001)$ in the rapamycin-treated group compared with DMSO (control) from day 4 to day 16 and in TIE2-TKI-treated group $(P<0.05)$ from day 6 to day 12 (Figure 3G). At day 15, vascular volume in the rapamycintreated group was lower compared with the DMSO and the TIE2TKI-treated groups $(P<0.001$ and $P<0.05$ respectively) (Figure $3 \mathrm{H})$.
Moreover, the vascular volume in the TIE2-TKI-treated group did not show a significant difference $(P=0.315)$ compared with controls.

We further examined the phenotype of the lesional blood vessels after TIE2-TKI and rapamycin treatment (following the schematic in Figure 3A). In response to rapamycin, blood vessels were smaller and showed an increased number of $\alpha \mathrm{SMA}^{+}$perivascular cells (Figure 3I). Rapamycin induced a significant decrease of vascular area compared with vehicle-treated and TIE2-TKI-treated mice $(P<0.01)$ (Figure 3J). Furthermore, the number of $\alpha \mathrm{SMA}^{+}$cells per vessel was significantly increased in response to rapamycin treatment compared with vehicle and TIE2-TKI treatment $(P \leq 0.01)$ (Figure 3K).

TIE2-TKI weakly inhibits TIE2 and AKT phosphorylation in TIE2-L914F cells compared with TIE2-WT and TIE2-R1099X. We investigated whether TIE2-TKI was effective at downregulating TIE2 signaling in vitro to gain insights into its weak suppression of VM growth. TIE2-TKI was tested in vitro at concentrations ranging from 0.5 to $10 \mu \mathrm{M}$ for activity on HUVEC-TIE2-WT, and TIE2 mutants HUVEC-TIE2-L914F and HUVEC-TIE2-R1099X. 
A

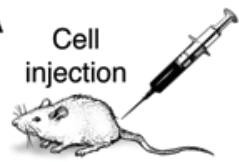

Day: 0

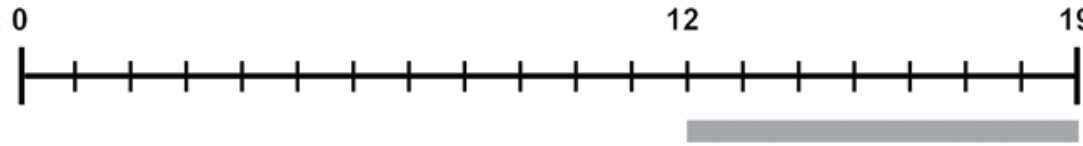

I.P. treatment -7 days

B

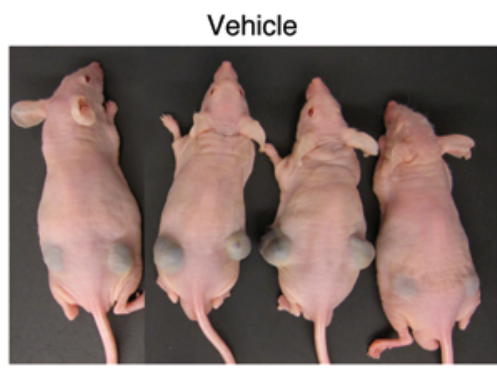

C
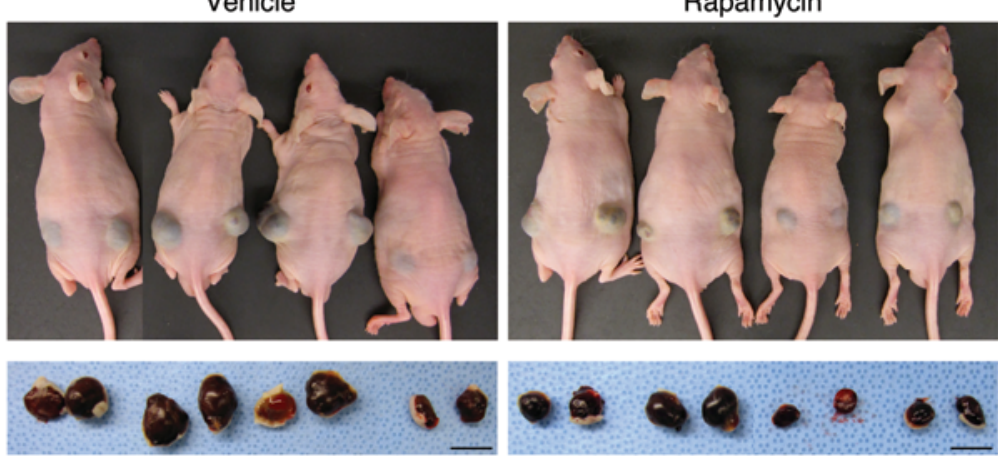

D

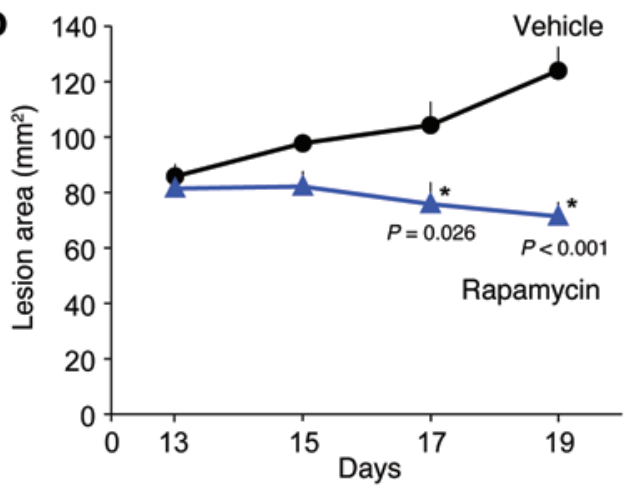

$\mathbf{F}$

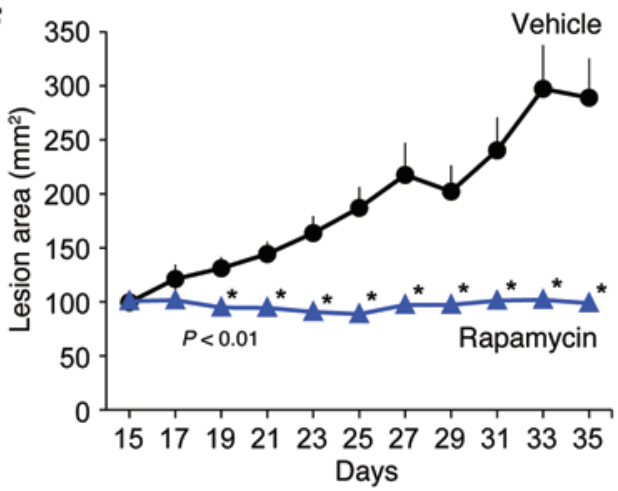

E

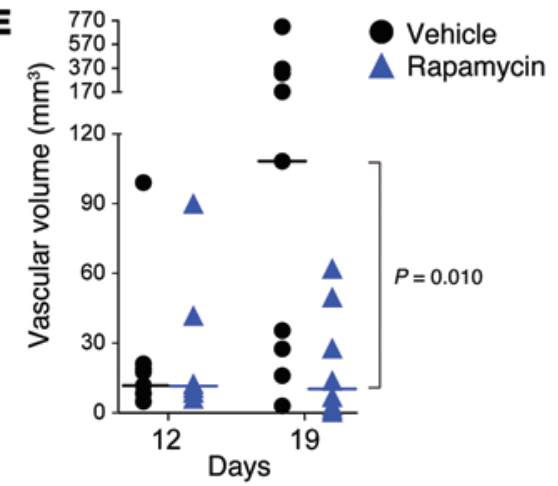

G

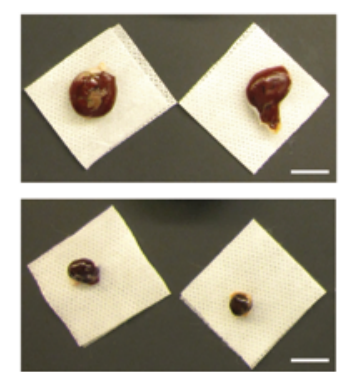

Vehicle

Rapamycin
Figure 6. Growth of established VMs was prevented by rapamycin treatment. (A) Treatment schematic. (B) Images of mice at day 19 after cell injection (day 7 after start of treatment). (C) Representative murine VM lesion explants after 7 days of i.p. treatment. Left panel: vehicle; right panel: rapamycin (1 $\mathrm{mg} / \mathrm{kg}$ ). Images taken from fixed distance. (D) HUVEC-TIE2-L914F lesional area measured by caliper every 2 days from day 13 to day 19 . Data expressed as mean \pm SEM, $t$ test ( $n=5$ mice with 2 lesions/ group). (E) Vascular volume of each lesion before (day 12) and after (day 19) treatment measured by analysis of color Doppler 3D image stacks. Data expressed as single values for each lesion and medians plotted as horizontal bars, 2-way repeated measures ANOVA. (F) HUVEC-TIE2L914F lesional area measured with calipers every other day from day 15 after cell injection (start of treatment, vehicle or rapamycin $2 \mathrm{mg}$ / $\mathrm{kg} / \mathrm{d}$ ) to day 35 (endpoint). Data are expressed as mean $+\mathrm{SEM}, t$ test ( $n=10$ mice with 2 lesions/group). From day 19 to day 35 , values in the 2 groups are statistically different. ${ }^{*} P<0.01, t$ test. (G) Representative murine VM lesion explant at day 35 after 20 days of vehicle or rapamycin treatment. Images taken from fixed distance. Scale bars: $1 \mathrm{~cm}$.
TIE2-R1099X is a nonsense mutation identified in VM patients (15). Located in the C-terminal domain of TIE2, R1099X mutation truncates the $\mathrm{C}$-terminal inhibitory loop and causes increased receptor autophosphorylation (15).

TIE2-TKI induced dose-dependent inhibition of phospho-TIE2 (p-TIE2) and phospho-AKT-Ser473(p-AKT473)/phospho-AKTThr308 (p-AKT308) in HUVEC-TIE2-WT, while it was less efficient in HUVEC-TIE2-L914F (Figure 4, A and B). HUVEC-TIE2-R1099X exhibited lower levels of $\mathrm{p}$-TIE2 compared with TIE2-L914F. There- fore the TIE2-TKI inhibition of p-TIE2 and p-AKT in HUVEC-TIE2R1099X was greater than in HUVEC-TIE2-L914F.

We further examined the ability of TIE2-TKI to suppress angiopoieitin-1-induced (ANGPT1-induced) signaling in HUVEC-TIE2WT versus mutant TIE2. TIE2-TKI effectively inhibited p-TIE2 and p-AKT473 induced by ANGPT1 stimulation of TIE2-WT-expressing and TIE2-R1099X-expressing HUVECs (Figure 4, C and D). In contrast, the high levels of p-TIE2 and p-AKT473 in ANGPT1-stimulated HUVEC-TIE2-L914F were not reduced by TIE2-TKI. 


\section{Table 2. Description of patients in the pilot study}

\begin{tabular}{|c|c|c|c|c|c|c|}
\hline Patient & 1 & 2 & 3 & 4 & 5 & 6 \\
\hline Sex & $\mathrm{F}$ & M & $\mathrm{F}$ & $\mathrm{F}$ & $\mathrm{F}$ & M \\
\hline Diagnosis & Sporadic VM & Sporadic VM & MSVM & Sporadic VM & KTS & Sporadic VM \\
\hline Genotype & No TIE2 mutation & TIE2 mutation (L914F) & TIE2 mutation (R1099X) & No TIE2 mutation & No TIE2 mutation & TIE2 mutation (L914F) \\
\hline
\end{tabular}

\section{Previous treatments}

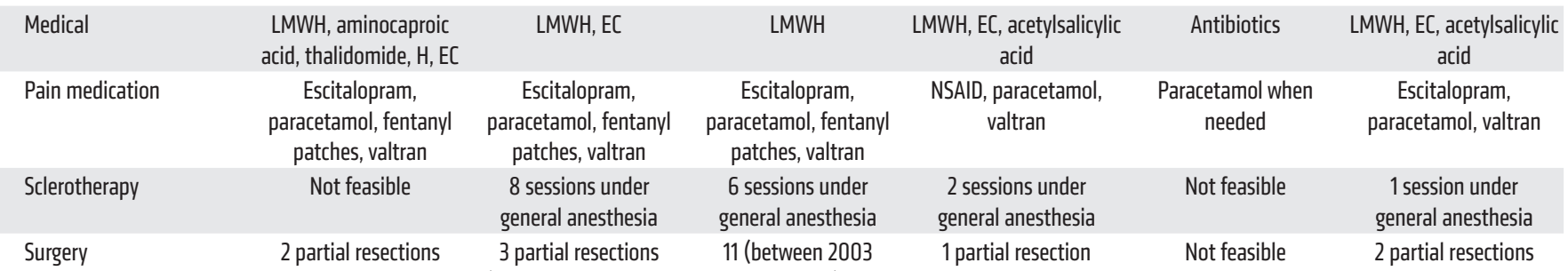

\section{Signs and symptoms}

$\begin{array}{lcccc}\text { Enlargement } & \begin{array}{c}\text { Thigh circumference } \\ 2.5 \mathrm{~cm}>\text { other side }\end{array} & \begin{array}{c}\text { Asymmetry of } \\ \text { the buttock }\end{array} & \text { None } & \text { Upper part of ankle } \\ \text { Functional } & \text { Muscular weakness, } & \text { Muscular weakness, } & \text { Muscular weakness, } & 0.5 \mathrm{~cm}>\text { other side } \\ \text { impairment } & \text { difficulties in } & \text { difficulties in walking, } & \text { difficulties in writing }>2 \mathrm{~h} \text {, } & \begin{array}{c}\text { Muscular weakness, } \\ \text { difficulties in }\end{array} \\ & \text { walking, sitting } & \text { sitting, standing still } & \text { doing sport (exercise) } & \text { standing }>2 \mathrm{~h}\end{array}$

\begin{tabular}{|c|c|c|c|c|c|c|}
\hline Pain (VAS) & 10 (daily for 8 years) & 8 (daily for 9 years) & 8 (daily for 4 years) & 8 (daily for 3 years) & $3-8$ (daily for 6 years) & 10 (daily for 2 years) \\
\hline Bleeding & Daily rectal & None & None & None & $\begin{array}{l}\text { Daily lesional bleeding } \\
\text { and oozing for } 10 \text { years }\end{array}$ & None \\
\hline Infection & None & None & None & None & $2 \times / \mathrm{mo}$ & None \\
\hline Other & Pulmonary embolism & None & None & None & $\begin{array}{l}\text { Chronic lesional wound, } \\
\text { scoliosis }\end{array}$ & None \\
\hline Self-perceived QoL & $\begin{array}{l}\text { Poor, severe mood } \\
\text { alteration }\end{array}$ & $\begin{array}{l}\text { Poor, severe mood } \\
\text { alteration, lost job }\end{array}$ & $\begin{array}{l}\text { Poor, severe mood } \\
\text { alteration }\end{array}$ & $\begin{array}{l}\text { Poor, unable to continue } \\
\text { current profession }\end{array}$ & $\begin{array}{l}\text { Poor, often absent } \\
\text { from school }\end{array}$ & $\begin{array}{l}\text { Poor, severe mood } \\
\text { alteration }\end{array}$ \\
\hline $\begin{array}{l}\text { Prior fibrinogen } \\
\text { values (mg/dl) } \\
\text { normal range: } 150-450\end{array}$ & $136 \pm 13(n=3)$ & $\begin{array}{c}170241 \pm 33 \\
\text { (on LMWH; } n=3 \text { ) }\end{array}$ & $248 \pm 16(n=4)$ & 438 & 371 & 212 and 209 \\
\hline $\begin{array}{l}\text { Prior D-dimer } \\
\text { values ( } \mathrm{ng} / \mathrm{ml} \text { ) } \\
\text { normal range: }<500 \\
n=\text { number of measurements }\end{array}$ & $8,060 \pm 212(n=3)$ & $\begin{array}{l}9,0033,491 \pm 375 \\
\text { (on LMWH; } n=3 \text { ) }\end{array}$ & $7,033 \pm 644(n=4)$ & 663 & 1,685 & 5,860 and 4,320 \\
\hline
\end{tabular}

Patients' general information, previous treatments, signs and symptoms, and prior biological values. If more than 2 measurements, mean \pm SEM is given; $n$, number of measurements. LMWH, low molecular weight heparin; $H$, heparin; EC, elastic compression.

Rapamycin treatment strongly inhibits $p-A K T 473$, bypassing the mutant TIE2. We next examined the capacity of rapamycin (15 $\mathrm{nM})$ compared with TIE2-TKI $(5 \mu \mathrm{M})$, to inhibit activation of the downstream target p-AKT $(36,37)$ in HUVECs expressing TIE2WT versus TIE2-L914F (Figure 5A).

As previously reported (ref. 30 and shown in Figure 4), a major effect of the elevated p-TIE2 in HUVEC-TIE2-L914F was strong activation of p-AKT. In TIE2-L914F, we detected increased p-AKT473 and p-AKT308, $38 \pm 0.067$-fold and $6 \pm 0.28$-fold higher than in HUVEC-TIE2-WT, respectively (Figure 5, A and B).
The TIE2-TKI treatment reduced p-TIE2 and p-AKT473 in HUVEC-TIE2-L914F, but the levels remained 18 and 21 times higher, respectively, than in the control HUVEC-TIE2-WT (DMSO treated) (Figure 5, A and B). Rapamycin treatment strongly inhibited p-AKT473 in HUVEC-TIE2-L914F, such that p-AKT 473 levels were reduced to a value $(1.415 \pm 0.552)$ comparable to TIE2-WT HUVECs $(1.000 \pm 0.378)$. In addition, both TIE2TKI and rapamycin mildly inhibited p-AKT308 in HUVEC-TIE2L914F, but did not significantly affect p-ERK levels. Rapamycin inhibition of p-AKT473 has been reported in endothelial cells in 
A

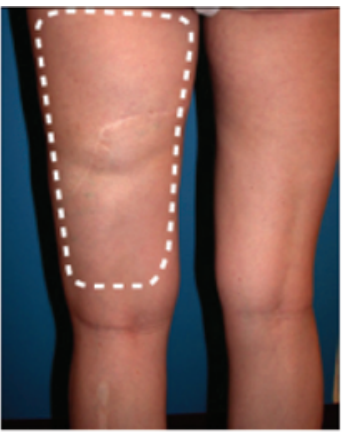

B

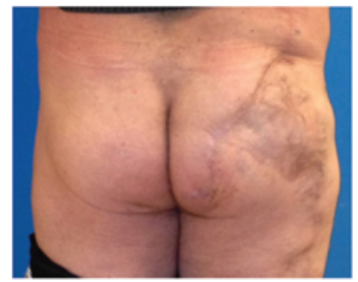

C

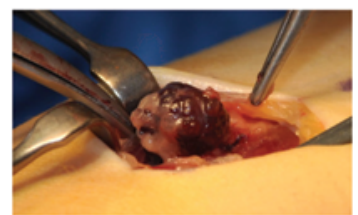

D

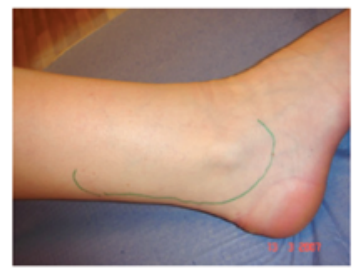

$\mathbf{E}$

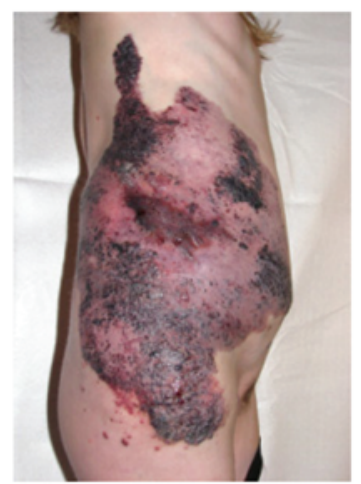

$\mathbf{F}$

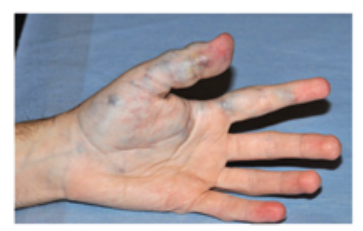

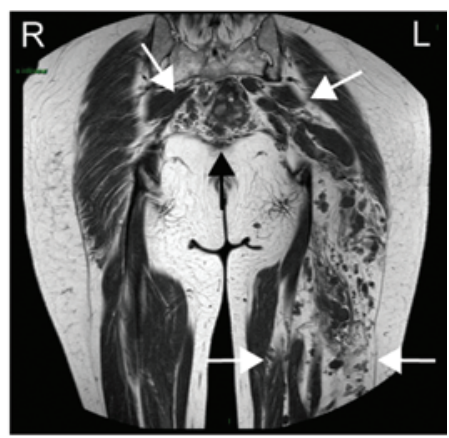
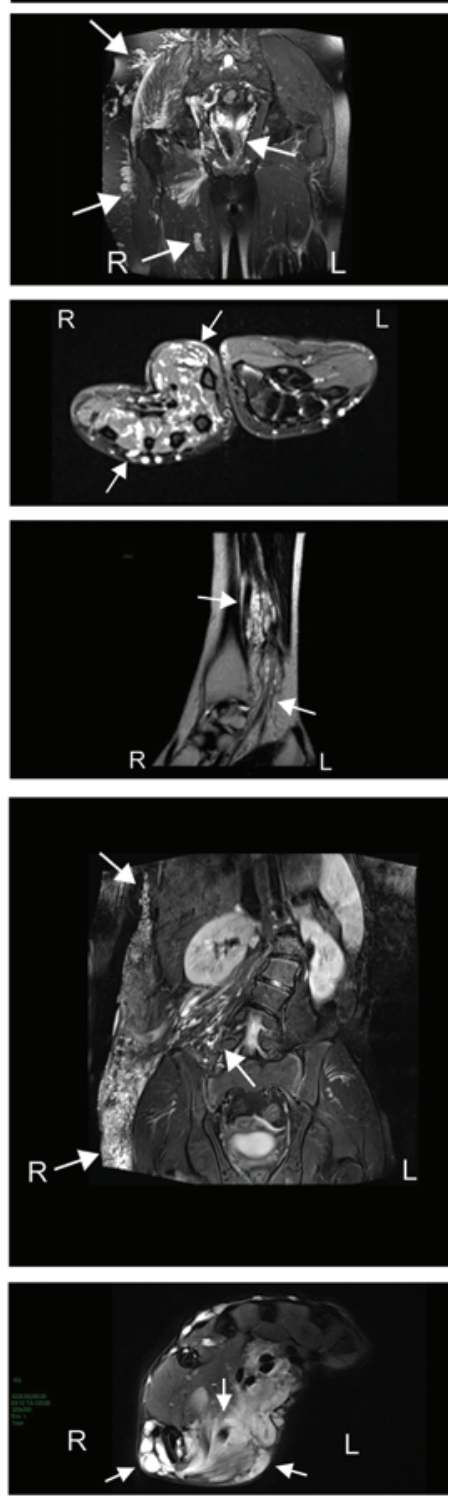

Figure 7. Clinical photographs and MRI images of patients in the pilot study. Clinical photographs (left panels) and MRI scans (right panels) of lesions in (A) patient 1; (B) patient 2; (C) patient 3, (D) patient 4; (E) patient 5; and (F) patient 6. L, left; R, right. Arrows point to lesions.

response to prolonged rapamycin treatment. This effect has been attributed to a rapamycin-mediated disruption of the mTORC2 complex that is responsible for the phosphorylation of AKT at Ser473 (38). p-4E-BP1, downstream of the mTORC1 pathway, did not show significant upregulation in the HUVEC-TIE2-L914F,

and levels of p-mTOR and p-4E-BP1 were mildly reduced by rapamycin treatment. Phosphorylation of STAT1 was higher in HUVEC-TIE2-L914F (30) compared with HUVEC-WT-TIE2. TIE2-TKI induced a reduction of p-STAT1/STAT1 levels, but rapamycin did not (Figure 5B).

Rapamycin prevents further expansion of established VMs in mice. In a third therapeutic scheme, untreated HUVEC-TIE2-L914F were implanted in mice and allowed to form VMs for 12 days; at this time, we started daily i.p. injections of rapamycin $(2 \mathrm{mg} /$ $\mathrm{kg}$ ) or vehicle for 7 days (Figure 6A). Lesions in mice treated with rapamycin were seemingly smaller in size compared with vehicle at day 19 (Figure 6, B and C) and showed significantly $(P<0.05)$ smaller area beginning at day 17 and continuing until day 19 (Figure 6D). In vehicle-treated mice, the vascular volume of lesions increased approximately 9 -fold from day 12 to day $19(P<0.01)$, while in rapamycin-treated mice there was no statistically significant increase $(P=0.958)$. At the end of treatment, the rapamycintreated group showed significantly $(P<0.05)$ smaller vascular volumes than the vehicle-treated group (Figure 6E). Prolonged rapamycin treatment, for 20 days, halted murine VM expansion, but was not sufficient to induce reduction in the size of the lesions (Figure 6, F and G).

Rapamycin alleviates clinical signs and symptoms in patients with VMs that are refractory to standard care. We investigated whether rapamycin could be effective in treating signs and symptoms in patients with VMs that could no longer be helped with standard therapies. Six patients, 4 females and 2 males, aged from 14 to 64 years (mean: 32 years) were enrolled in the study (Table 2 and Figure 7). All patients had severe symptoms despite several sequences of earlier sclerotherapies and/or surgical resections. The signs and symptoms varied and included disfigurement $(n=5)$, pain despite large doses of pain killer medications $(n=6)$ (visual analogue scale [VAS] between 3 and 10), functional impairment $(n=6)$, bleeding $(n=2)$, recurrent infections necessitating hospitalization with i.v. antibiotics $(n=1)$, and oozing and congestion $(n=1)$. All patients had elevated D-dimers (from 663 to $12,200 \mathrm{ng} / \mathrm{ml}$ ). One of them also had abnormally low fibrinogen levels $(136 \pm 13 \mathrm{mg} / \mathrm{dl})$ (Table 2).

All patients clinically improved with rapamycin (Table 3, Figure 8 , and Supplemental Table 3). Follow-up varied from 3 months to 16 months (mean: 12 months), with all but 1 patient having completed 12 months of follow-up. After the first 3 months of medication, patient no. 1 stopped bleeding. Her painful episodes decreased in intensity (VAS from 10 to 8) and in frequency (from twice a week to once a month). Her quality of life (QoL) increased by $30 \%$. The volume of her affected leg normalized (compared with the contralateral side), and she no longer presented difficulties in dressing or walking. Patient no. 2 had a reduction of the swelling of his malformation and less pain, with VAS evolving from 8 to 1 . He could also sit longer than 2 hours without pain. His mobility increased and QoL ameliorated by $80 \%$. Patient no. 3 had a drop of pain from VAS 8 to 2 . Patient no. 4 no longer needed elastic compression in daily work as a nurse, and her pain level dropped from VAS 8 to 1 . Moreover, her QoL ameliorated by $90 \%$. Patient no. 5 had less bleeding and oozing from her lesion, and infections subsided. Patient no. 6 experienced improved mobility of his wrist and thumb, and pain reduced from VAS 10 daily to 8 twice a month. 
Table 3. Evolution of quantitative parameters in 6 patients under rapamycin therapy

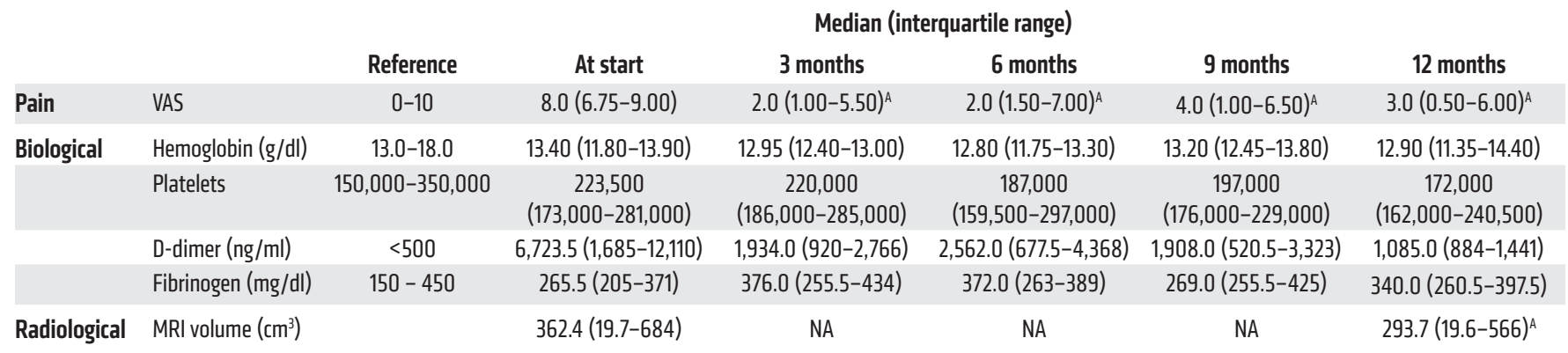

Values calculated on single measures for each patient at each time point; at start and 3 months, 6 patients; at 6 , 9 , and 12 months, 5 patients. ${ }^{A} P<0.05$ vs. start, Wilcoxon's signed-rank test. NA, not applicable.

At 6 and 12 months, 5 patients were still on medication and continued to have improved function and less bleeding, oozing, and pain (Tables 2 and 3). Patient no. 6 stopped medication due to grade 3 mucositis. Patient no. 1 had stopped rapamycin for 1 week because of fatigue and grade 2 headaches, but restarted the therapy, as vaginal and rectal bleedings had reoccurred. The bleedings stopped again after 24 hours of reinitiation of the medication. Patient no. 2 witnessed resurrection of pain almost at pretreatment level at 6 months of treatment. Rapamycin was stopped for 2 weeks. Functional impairment recurred, and pain increased. Rapamycin was reintroduced and pain reduced to VAS 1 at the 12-month time point. Rapamycin dosage of patient no. 3 was increased to $3 \mathrm{mg} / \mathrm{d}$ due to low serum levels. Patient no. 4 had become asymptomatic. Patient no. 5 halted rapamycin treatment for 4 weeks after 10 months on medication because she had to have surgical correction of her scoliosis. After reintroduction of rapamycin, her pain and D-dimers returned to the earlier reduced levels.

In general, VAS scores improved significantly $(P<0.05)$ over time, reflecting decreased pain experienced by all patients (Table 3, Figure 8, and Supplemental Table 3). Moreover, a statistically significant reduction $(P<0.05)$ in $\mathrm{VM}$ volume was observed, with MRI measurements showing a decrease in all the 5 patients that reached 12 months follow-up (362.4 [19.7684] $\mathrm{cm}^{3}$ versus 293.7 [19.6-566] $\mathrm{cm}^{3}$ ) (Table 3, Figure 8, and Supplemental Table 3). Additional parameters showed marked improvements across patients (Table 3 and Supplemental Table 3), albeit statistically inconclusive due to the limited sample size. Biological parameters were as follows: D-dimer levels dropped (from 6,723.5 [1,685-12,110] ng/ml to $1,085.0$ [884-1,441] ng/ $\mathrm{ml}$ ) between the initiation of therapy (start) and the 12-month time point (Table 3 and Figure 8). Fibrinogen levels normalized in the 1 patient in whom they were abnormal and increased in all patients except patient 4 (from 265.5 [205-371] mg/dl to 340.0 [260.5-397.5] mg/dl) over the same time period. Esthetic and functional impairment decreased in all patients. In patients no. 1 and no. 5 bleeding stopped, and patient no. 5 had no more lesional infections. Finally, patients' assessments of improvement in QoL relative to the start of treatment ranged from 30\% to $90 \%$ by 3 months (median $70 \%, n=6$ ) and $30 \%$ to $100 \%$ by 12 months (median 90\%, $n=5$ ) (Supplemental Table 3).
All new signs and symptoms that appeared during the clinical trial were recorded as possible side effects, according to the NIH Common Terminology Criteria for Adverse Events (CTCAE), version 3, classification. Two such possible side effects were recorded as "severe." Patient no. 1 developed a malignant cutaneous tumor after 1 year on treatment (grade 4 basal cell carcinoma). It was cured by surgical resection. Patient no. 6 had grade 3 mucositis, necessitating cessation of the rapamycin treatment. All other signs and symptoms were of the minor category and consisted of grade 1 or 2 headache $(n=5$ and $n=1)$, rash $(n=3)$, grade 1 or 2 fatigue $(n=2$ and $n=2)$, grade 1 or 2 mucositis $(n=1$ and $n=2)$, cutaneomucosal dryness $(n=2)$, grade 2 flu-like syndrome $(n=1)$, grade 1 neuropathy $(n=1)$, urinary trouble $(n=1)$, edema $(n=1)$, diarrhea $(n=1)$, rhinorrhea $(n=1)$, vomiting $(n=1)$, thoracic pain $(n=1)$, dizziness $(n=1)$, and arthralgia $(n=1)$.

\section{Discussion}

We report here what we believe is the first model of human $\mathrm{VM}$ in mice, the therapeutic effect of rapamycin in this model, and a clinical response to rapamycin in 6 patients affected by VMs that were refractory to standard care. The murine VM model establishes that endothelial cells engineered to express the most frequent TIE2 mutation (L914F) associated with sporadic VMs $(14,15)$ form VM lesions in mice within a few days, with enlargement occurring over time. Efficacy of rapamycin in this model and in patients in our clinical pilot study suggests rapamycin as the first molecular therapy for VMs.

The Tie2 receptor, activated by ligand binding, signals via the PI3K/AKT pathway $(36,37)$. Tie2 autophosphorylation is negatively regulated by the $\mathrm{C}$-terminal domain of the receptor $(39,40)$, and deletion of Tie $2 \mathrm{C}$-terminal 16 amino acids results in Tie2 hyperphosphorylation and enhanced AKT signaling (39). Similar truncating, somatic mutations have been identified in sporadic VMs (including patient no. 3 in the current study) (15). The L914F mutation, like all other reported VM-causing TIE2 kinase domain substitutions, also elicits TIE2 receptor hyperphosphorylation and enhanced AKT signaling in cultured endothelial cells (30). Sustained endothelial AKT activation caused vascular malformations and increased blood vessel size in a transgenic myristoylated AKT mouse model (35). Rapamycin 

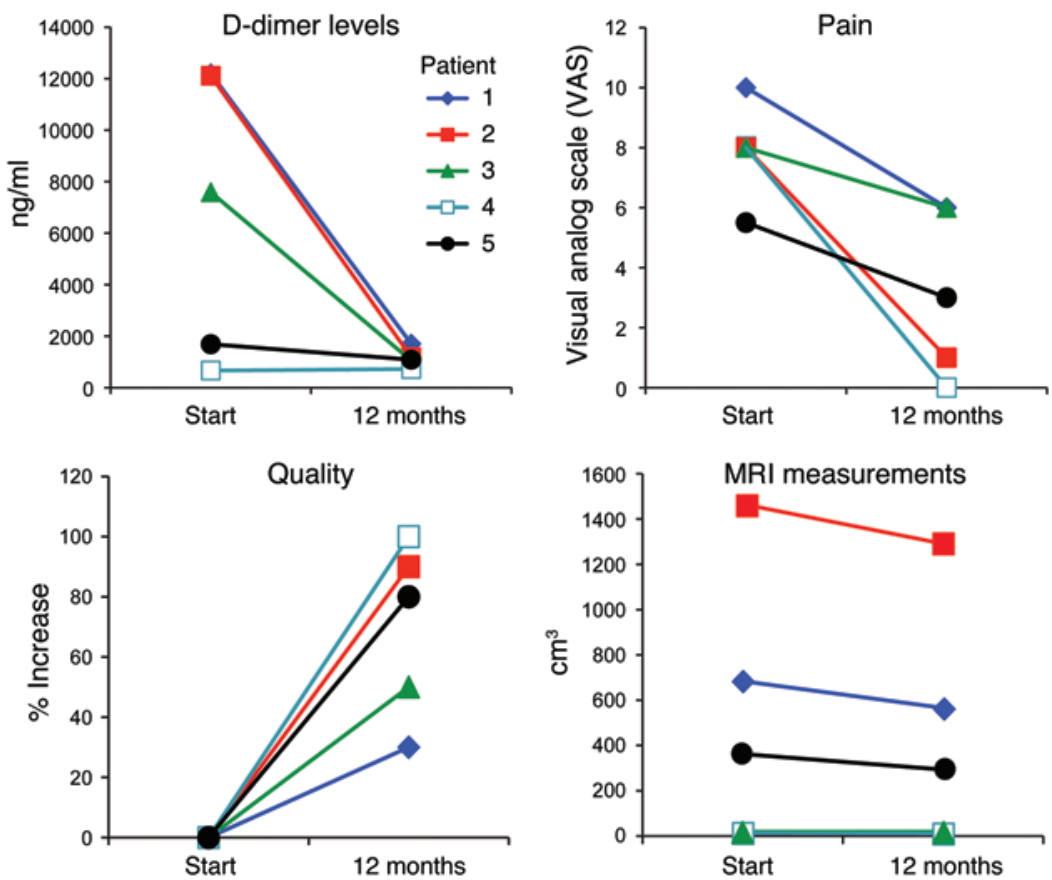

Figure 8. Evolution of quantitative parameters from start to 12 months after rapamycin treatment. Analysis of D-dimer levels, pain, QoL, and MRI measurements in 5 patients at start and after 12 months of rapamycin treatment.

blocked and even reverted pathological blood vessel formation and reduced p-AKT levels in this model (35). These findings link TIE2 mutations and activation of AKT to enlarged vascular channels that are the hallmark of VM.

There are currently no targeted pharmacological therapies for VM $(3,4)$. The murine VM model we report here provides a platform to test candidate drugs for the ability to prevent mutant TIE2-dependent VM formation and enlargement. Rapamycin, an FDA-approved drug first used to suppress $\mathrm{T}$ cell proliferation, inhibits the MTOR pathway and prevents proliferation and protein synthesis in many types of mammalian cells (41, 42). Rapamycin first targets mTORC1, yet chronic exposure of endothelial cells to rapamycin can disrupt the mTORC2 complex (43), which is responsible for the phosphorylation of AKT at Ser473 (38). In our murine model, rapamycin reduced the growth of VM lesions in 3 different treatment schemes, while TIE2-TKI was less potent.

Our molecular in vitro studies determined that TIE2-TKI treatment of L914F HUVECs decreased phosphorylation of TIE2 only weakly and therefore modestly reduced the activation of AKT. The TIE2-TKI compound has been designed to potently inhibit the TIE2-WT kinase (33). Our results indicate that it has a reduced ability to target the L914F mutant TIE2. The negative regulation of TIE2 autophosphorylation is maintained by a salt bridge between Arg914 (915 in human) and C-terminal tail glutamate 1120 in murine Tie2 $(39,40)$. We postulate that the increased TIE2 activation in the L914F mutant is due to the disruption of this autoinhibitory receptor conformation (Leu914 being adjacent to Arg915 in a highly conserved region). A change in protein conformation could cause the low efficiency of TIE2TKI on TIE2-L914F we report here. HUVEC-TIE2-R1099X showed an inhibitory response to TIE2-TKI, similar to TIE2-WT, again suggesting TIE2-TKI activity is sensitive to specific TIE2 mutations and resulting conformations. In contrast, rapamycin reduced AKT phosphorylation at Ser473 (likely through sustained sequestration of $\mathrm{mTOR}$ and dismantlement of the mTORC2 complex; ref. 38) in HUVEC-TIE2L914F, normalizing the p-AKT levels to near that of HUVEC-TIE2-WT. Because rapamycin inhibits AKT signaling downstream of TIE2, it is able to "bypass" the mutant TIE2.

Rapamycin is currently being tested in a multicenter clinical trial on lymphatico-vascular malformations (NCT00975819; www.clinicaltrials.gov) (44). In 2 independent studies, low-dose rapamycin $(0.05-0.1 \mathrm{mg} / \mathrm{kg})$ resulted in successful treatment of blue rubber bleb nevus syndrome (45) and in clinical improvement in children with complicated vascular anomalies (46). We initiated a clinical pilot study to test rapamycin on VMs that are refractory to standard care on the basis of the activation of the PI3K/AKT/ mTOR pathway downstream of TIE2 mutations. Half of the patients had a somatic TIE2 mutation (Supplemental Table 4). The other half likely activated the same signaling pathway via mutations in a protein that closely interacts with TIE2 or the downstream signaling pathway. On the basis of all the preclinical data, we postulated that VM patients would respond to rapamycin.

Our clinical pilot study strongly suggests that rapamycin can reduce VM patients' signs and symptoms. Within the first month of rapamycin administration, all 6 patients experienced a relief of pain, better function of the affected body part, and improved selfperceived QoL. Rapamycin had an on/off effect on bleeding and oozing. Biological markers (D-dimers and fibrinogen) improved, and MRI images showed significant $(P<0.05)$ decrease in volume after 12 months on treatment. Some major and minor signs and symptoms, which may be side effects of rapamycin, were recorded during therapy. Therefore, it is important to restrict inclusion of patients in rapamycin trials and to have a careful follow-up of the patients. Overall, in this cohort, benefits outweighed side effects in patients with VMs that were refractory to standard care.

This is the first report, to our knowledge, of an effective molecular therapy for VM. As rapamycin is likely a life-long treatment with probable side effects, it should not be considered as a treatment for small, localized, painless, nonbleeding VMs that are amenable to standard care. As it is unclear whether the improvements reported in these patients can be generalized to all patients with difficult-to-treat VMs, continued rigorous and systematic study of the safety and efficacy of rapamycin for the treatment of VM is required. We also need to assess long-term outcomes. Additional laboratory work is also necessary to define the exact cellular mechanism or mechanisms by which rapamycin affects VMs. It is interesting to speculate that, in patients with TIE2 mutations sensitive to TIE2-TK inhibition, such as R1099X, combination therapy with rapamycin and an inhibitor directed at the TKD of TIE2 could induce a greater patient clinical improvement than rapamycin alone. 


\section{Methods}

Cell culture. Retrovirally transfected HUVECs expressing full-length TIE2-WT, TIE2-L914F, or TIE2-R1099X were expanded in culture on attachment factor-coated dishes and fed with Endothelial Cell Growth Medium (Cell Applications)/10\% FBS (HyClone). HUVECs were provided by F.W. Luscinskas (Brigham and Women's Hospital, Boston, Massachusetts, USA).

In vivo murine model of VM. $2.5 \times 10^{6}$ HUVECs (TIE2-WT or TIE2L914F) were suspended in Matrigel (Corning) and injected s.c. into the lower backs of 6- to 7-week-old male athymic nu/nu mice (Massachusetts General Hospital) ( $n=10 /$ group) as described (47). Area of lesions $\left(\mathrm{mm}^{2}\right)$ was measured with caliper. For drug studies, HUVEC-TIE2L914F were incubated for 48 hours in vitro with $5 \mu \mathrm{M}$ TIE2-TKI (EMD Millipore), $15 \mathrm{nM}$ rapamycin (LC Laboratories), or DMSO as control. Animals received daily i.p. injection of $200 \mu$ l vehicle, TIE2-TKI (10 $\mathrm{mg} / \mathrm{kg})$, or rapamycin $(2 \mathrm{mg} / \mathrm{kg})$. Blood gases were measured on an ABL800 FLEX analyzer (Radiometer Medical). Blood cell counts were obtained from an ADVIA 2120 Hematology Analyzer (Siemens).

Color Doppler ultrasonography. Vascular volume and blood flow were measured using the Vevo 2100 High-Resolution Ultrasound System (Visual Sonics). Analysis of flow velocity and sound (venous versus arterial) was performed in pulse-waved Doppler mode. Images were acquired, via color Doppler mode, at $100 \mu \mathrm{m}$ intervals from the anterior to posterior ends of the lesions. The images were assembled to form a 3D image stack. To analyze 3D data, the perimeter of the construct was drawn on serial slices 0.5 to $1 \mathrm{~mm}$ apart. The software defined the volume and percentage of vascular volume by interpolating between the regions of interest.

$V D$. For the assessment of vessels $/ \mathrm{mm}^{2}$ (VD), human CD $31^{+}$luminal structures containing red blood cells were counted in 10 fields/ section. Vascular area in 10 fields/explant was measured with Image J software (http://imagej.nih.gov/ij/).

Immunohistochemistry and immunofluorescence. Paraffin sections of murine VM explants were stained with anti-human CD31 (clone JC70-A, Dako) or with biotinylated UEA-I (Vector Labs) to detect human endothelial cells and with anti- $\alpha$ smooth muscle actin ( $\alpha$ SMA) (clone 1A4, Sigma-Aldrich) to detect smooth muscle/ perivascular cells. This was followed by peroxidase secondary antibody, FITC secondary antibody, or Texas red-streptavidin for UEA-I (Vector Labs). Nuclei were stained with hematoxylin or DAPI (Life Technologies).

Immunoblots. Cell lysates were analyzed by Western blot with antibodies against the following: p-mTOR (clone D9C2), mTOR (catalog 2972), p-AKT (Ser473 and Thr308, clone 587F11 and C31E5E, respectively), AKT (catalog 9271), p-4E-BP1 (Ser65), 4E-BP1 (catalog 9452), p-ERK (Thr202/Tyr204), ERK (no. 9102), p-STAT1 (clone D4A7), STAT1 (catalog 9172) (all from Cell Signaling Technology); p-TIE2 (clone Ab-1, Millipore); TIE2 (clone 16, Abcam); tubulin (clone DM1A, Sigma-Aldrich), and $\beta$-actin (clone AC-15, Sigma-Aldrich).

Patients. Only patients with a symptomatic venous or combined slow-flow malformation that was refractory to standard care, such as analgesic medication, low-molecular weight heparin, elastic compression, sclerotherapy, and/or surgical resection, were included in the study. There was no age limitation. Patients needed to have normal hepatic function (alanine transaminase [ALT], aspartate transaminase [AST], $\gamma$-glutamyl transpeptidase $[\gamma \mathrm{GT}]$, LDL cholesterol, and triglycerides), medullary function (neutrophils $>1500 / \mathrm{mm}^{3}$, hemoglobin $>8.0 \mathrm{mg} / \mathrm{dl}$, and platelets $>50,000 / \mathrm{mm}^{3}$ ), and renal function (creatinine in function of age, creatinine clearance $>70$ $\mathrm{ml} / \mathrm{min} / 1.73 \mathrm{~m}^{2}$, and a urine protein/creatinine ratio [UPC] $<0.3 \mathrm{~g}$ ) with a Karnofsky or Lansky index greater than 50. Exclusion criteria were based on the following: (a) severe concurrent and/or not controlled disease (e.g., diabetes, infection, HIV, hypertension), (b) use of CYP3A4 inhibitors/inducers, (c) gastrointestinal disorders, which might modify absorption of rapamycin, and (d) pregnancy or breast feeding. On this basis, 6 patients were included. Four patients had a single large sporadic VM, 1 had a multifocal sporadic venous malformation (MSVM), and 1 had a complex combined capillary-lymphatico-venous malformation with overgrowth, also known as KlippelTrenaunay syndrome (KTS) (Table 1).

Toxicity was evaluated according to CTCAE, version 3 (http:// ctep.cancer.gov/protocoldevelopment/). This was performed at each consultation, weekly during the first month, monthly during the second and third months of trial, and then every 3 months. If a patient had any new clinical signs or symptoms, he or she was asked to contact the study coordinator to organize an in-between evaluation.

Treatment was suspended in cases of (a) severe toxicity (CTCAE grade 4 ) and (b) when milder side-effects (grade $\leq 3$ ) were not acceptable for the patient and/or parents, and (c) patient did not experience benefit from medication after 6 months of therapy. Patients left the protocol in the case of loss from medical monitoring, refusal of the follow-up protocol, or death. If complete response to rapamycin was observed, treatment was suspended and the patient followed for a total of 5 years.

Study protocol. The clinical trial was started in 2012. Before initiation of rapamycin treatment, patients were examined by the coordinator of the multidisciplinary Center for Vascular Anomalies (L.M. Boon, plastic surgeon). Diagnosis was based on clinical history and aspect of the lesion (color, consistency, temperature compared with contralateral side) as well as biological (D-dimer and fibrinogen levels) and radiological analyses (Doppler ultrasound and MRI) $(3,4)$. Specific clinical signs and symptoms related to the venous malformation were evaluated and marked, including pain with VAS (ranging from $0-10 ; 0=$ no pain), location of the lesion, enlargement of the affected body part, and evaluation of the patient's functional impairment (Table 1). Psychological impact of the malformation was evaluated using a QoL questionnaire (modified from the RAND MOS SF-36 survey) (48) and a global self-evaluation of percentage of increase in QoL (scale: $0 \%=$ no change to $100 \%=$ symptom free). Adult or pediatric oncologists (E. Seront and S. Dupont) also examined the patient before initiation of rapamycin in order to exclude any contraindication and to explain the study protocol and the possible side effects of the medication. Biological evaluation (platelet counts, D-dimer and fibrinogen levels, hepatic, renal, and bone marrow functions), MRI imaging (T1, T2, and fat-sat sequences in 2 orthogonal planes), volume measurement using ITK-SNAP ${ }_{3-0}$, and clinical photographs of the visible lesions were done before initiation of the study. MRI study with volume measurement was repeated at 12 months or at cessation of therapy (at 3 months for patient no. 6).

Rapamycin was started with a single dose of $2 \mathrm{mg} / \mathrm{d}$. The serum target concentration was 10 to $15 \mathrm{ng} / \mathrm{ml}$. Rapamycin was dose adjusted (1-3 mg/d) (according to weekly serum concentrations) until 2 stable measurements at the aimed level were obtained. The serum level was subsequently checked every 3 months unless dosage had to 
be adjusted, in which case it was remeasured a week after the adjustment. Patients were seen after 1 week, then monthly for a total of 3 months, and finally every 3 months by both the plastic surgeon and the oncologist in order to evaluate the signs and symptoms of the malformation, compliance, and possible side effects of the drug.

Targeted deep sequencing of TIE2-coding region. The 23 coding exons of TIE2 (and 10 flanking nucleotides on either side of each; human genome assembly GRCh37/hg19) were included in an Ion AmpliSeq Custom DNA Panel (Life Technologies), which consists of pools of primers for multiplexed PCR amplification of regions of interest, followed by targeted sequencing on the Ion Personal Genome Machine (PGM) sequencer (Life Technologies). Detailed protocols are available in the Supplemental Methods. Targeted deep sequencing of TEK was carried out on 6 tissue DNAs (patients 1-6) and 5 blood DNAs (patients 2-6) (Supplemental Methods). Aligned sequence (.bam) files have been submitted to the European Nucleotide Archive (www.ebi. ac.uk) (ID PRJEB969).

Statistics. For the animal experiments, data were expressed as mean \pm SEM and analyzed by ANOVA followed by Student's $t$ test after normal distribution was assessed. When values did not follow normal distribution, data were plotted as single values and medians and analyzed with Mann-Whitney $U$ test or Wilcoxon's signed-rank test (for paired measurements). Two-way repeated-measures ANOVA was used to account for 2 VM lesions from the same mouse, and each mouse was evaluated at 2 different time points (days 12 and 19). For each group studied, $n=8-10$ animals or $n=5$ animals with 2 lesions on each side. Differences were considered statistically significant at $P<0.05$.

For the clinical parameters, statistical analyses were performed using the SPSS package, version 20 (IBM). Baseline characteristics of the patients are listed per patient and summarized as mean \pm SEM per patient in cases in which 3 or more prior measurements were available (Table 1). For parameters for which 3 or more numerical time points were available from the start of treatment (VAS, hemoglobin, platelets, D-dimers, fibrinogen), the Friedman repeated-measures test was used to test for overall differences in distribution over time (start, 3, 6, 9, and 12 months) (Table 2 and Supplemental Table 3). Where a significant difference $(P<0.05)$ was observed, post-hoc pairwise tests (Wilcoxon's signed-rank) were applied to detect significant differences among all combinations of paired time points. Due to the exploratory nature of the study (hypothesis generating vs. confirmatory), no corrections for multiple testing were applied. Where only 2 time points were available ( 0 months vs. 12 months, lesion volume by MRI), Wilcoxon's signed-rank test was used. Missing values (patients 4 and 6) could be assumed as unrelated to patient condition and outcome and were therefore omitted as missing completely at random (MCAR data).

Study approval. All animal studies were conducted in accordance with the NIH guidelines for humane treatment of animals and were approved by the Institutional Animal Care and Use Committee at
Boston Children's Hospital (OLAW assurance no. A3303-01). The Biomedical Ethical Committee of Cliniques Universitaires Saint Luc, Université Catholique de Louvain, approved the protocol of the patient study. Each patient (or patient's parents) signed an informed consent form and received a patient information form, adapted to the patient's age, and a summary explaining the procedure of the study. The trial is registered under the protocol ID VASC-LM at clinicaltrials. gov (NCT01811667). Patients (and parents of patients under 18 years of age) provided written, informed consent for publishing the clinical photographs and MRIs.

\section{Acknowledgments}

Research reported in this manuscript was supported by the National Institute of Arthritis and Musculoskeletal and Skin Diseases (P01 AR0 48564 to J. Bischoff and M. Vikkula) and by the National Heart, Lung, and Blood Institute(R01 HL117952 to E. Boscolo). The content is solely the responsibility of the authors and does not necessarily represent the official views of the NIH. Additional support was from the Manton Center for Orphan Disease (to E. Boscolo) and the Charles H. Hood Foundation (to E. Boscolo), the Belgian Science Policy Office Interuniversity Attraction Poles (BELSPO-IAP) Programme through the project IAP P7/43-BeMGI (to M. Vikkula), the F.R.S.-FNRS T.0026.14 (Fonds de la Recherche Scientifique) (to M. Vikkula), and the Academy of Finland (136880 and Centre of Excellence Program 2012-2017 to L. Eklund). J. Soblet was supported by a fellowship from Télévie, Belgium. We also acknowledge the support of la Communauté Française de Wallonie-Bruxelles and la Lotterie Nationale, Belgium. We thank John B. Mulliken, Harry Kozakewich, Harriet Paltiel, David Zurakowski, Basilia Zingarelli, and Anita Gupta for their expert advice and Frederick R. Roberts for technical assistance with the Vevo system (VisualSonics). We thank David Smadja, Stacy Croteau, Giulia Ciaghi, Jill Wylie Sears, and Jaana Träskelin for insightful discussions and technical help, the Dana-Farber/Harvard Cancer Center (DF-HCC) for Specialized Histopathology (HSP) Core, Kristin Johnson for the preparation of figures, and Jennifer Hammer for help in registering the clinical trial.

Address correspondence to: Elisa Boscolo, Cancer and Blood Disease Institute, Cincinnati Children's Hospital Medical Center, 3333 Burnet Avenue, MLC 7015, Cincinnati, Ohio 45229-3039, USA. Phone: 513.803.7267; E-mail: elisa.boscolo@cchmc.org.

Elisa Boscolo's present address is: Cancer and Blood Disease Institute, Cincinnati Children's Hospital Medical Center, Cincinnati, Ohio, USA.

Kyu-Tae Kang's present address is: College of Pharmacy, Duksung Women's University, Seoul, South Korea.
1. Boon LM, Mulliken JB, Enjolras O, Vikkula M. Glomuvenous malformation (glomangioma) and venous malformation: distinct clinicopathologic and genetic entities. Arch Dermatol. 2004;140(8):971-976.

2. Brouillard P, Vikkula M. Genetic causes of vascu- lar malformations. Hum Mol Genet. 2007; 16 Spec No. 2:R140-R149.

3. Burrows PE, Mason KP. Percutaneous treatment of low flow vascular malformations. J Vasc Interv Radiol. 2004;15(5):431-445.

4. Dompmartin A, Vikkula M, Boon LM. Venous malformation: update on aetiopathogenesis, diagnosis and management. Phlebology. 2010;25(5):224-235.

5. Dompmartin A, et al. Association of localized intravascular coagulopathy with venous malformations. Arch Dermatol. 2008;144(7):873-877. 
6. Dompmartin A, et al. Elevated D-dimer level in the differential diagnosis of venous malformations. Arch Dermatol. 2009;145(11):1239-1244.

7. Mazoyer E, Enjolras O, Bisdorff A, Perdu J, Wassef M, Drouet L. Coagulation disorders in patients with venous malformation of the limbs and trunk: a case series of 118 patients. Arch Dermatol. 2008;144(7):861-867.

8. Oduber CE, Gerdes VE, van der Horst CM, Bresser P. Vascular malformations as underlying cause of chronic thromboembolism and pulmonary hypertension. J Plast Reconstr Aesthet Surg. 2009;62(5):684-689.

9. Hammer FD, Boon LM, Mathurin P, Vanwijck RR Ethanol sclerotherapy of venous malformations: evaluation of systemic ethanol contamination. J Vasc Interv Radiol. 2001;12(5):595-600.

10. Hein KD, Mulliken JB, Kozakewich HP, Upton J, Burrows PE. Venous malformations of skeletal muscle. Plast Reconstr Surg. 2002;110(7):1625-1635.

11. Marrocco-Trischitta MM, Guerrini P, Abeni D, Stillo F. Reversible cardiac arrest after polidocanol sclerotherapy of peripheral venous malformation. Dermatol Surg. 2002;28(2):153-155

12. Mason KP, Neufeld EJ, Karian VE, Zurakowski D, Koka BV, Burrows PE. Coagulation abnormalities in pediatric and adult patients after sclerotherapy or embolization of vascular anomalies. AJR Am J Roentgenol. 2001;177(6):1359-1363.

13. Vikkula M, et al. Vascular dysmorphogenesis caused by an activating mutation in the receptor tyrosine kinase TIE2. Cell.1996;87(7):1181-1190.

14. Limaye N, et al. Somatic mutations in angiopoietin receptor gene TEK cause solitary and multiple sporadic venous malformations. Nat Genet. 2009;41(1):118-124.

15. Soblet J, Limaye N, Uebelhoer M, Boon LM, Vikkula M. Variable somatic TIE2 mutations in half of sporadic venous malformations. Mol Syndromol. 2013;4(4):179-183.

16. Wouters V, et al. Hereditary cutaneomucosal venous malformations are caused by TIE 2 mutations with widely variable hyper-phosphorylating effects. Eur J Hum Genet. 2010;18(4):414-420.

17. Calvert JT, et al. Allelic and locus heterogeneity in inherited venous malformations. Hum Mol Genet. 1999;8(7):1279-1289.

18. Augustin HG, Koh GY, Thurston G, Alitalo K. Control of vascular morphogenesis and homeostasis through the angiopoietin-Tie system. Nat Rev Mol Cell Biol. 2009;10(3):165-177.

19. Eklund L, Saharinen P. Angiopoietin signaling in the vasculature. Exp Cell Res. 2013;319(9):1271-1280.

20. Arai F, et al. Tie2/angiopoietin-1 signaling regulates hematopoietic stem cell quiescence in the bone marrow niche. Cell. 2004;118(2):149-161. 21. De Palma M, et al. Tie2 identifies a hematopoi- etic lineage of proangiogenic monocytes required for tumor vessel formation and a mesenchymal population of pericyte progenitors. Cancer Cell. 2005;8(3):211-226.

22. Davis $S$, et al. Isolation of angiopoietin-1, a ligand for the TIE2 receptor, by secretion-trap expression cloning. Cell. 1996;87(7):1161-1169.

23. Dumont DJ, Yamaguchi TP, Conlon RA, Rossant J, Breitman ML. tek, a novel tyrosine kinase gene located on mouse chromosome 4 , is expressed in endothelial cells and their presumptive precursors. Oncogene. 1992;7(8):1471-1480.

24. Sato TN, Qin Y, Kozak CA, Audus KL. Tie-1 and tie-2 define another class of putative receptor tyrosine kinase genes expressed in early embryonic vascular system. Proc Natl Acad Sci US A. 1993;90(20):9355-9358.

25. Suri C, et al. Requisite role of angiopoietin-1, a ligand for the TIE2 receptor, during embryonic angiogenesis. Cell.1996;87(7):1171-1180.

26. Dumont DJ, Gradwohl G, Fong GH, Puri MC Gertsenstein M, Auerbach A, Breitman ML. Dominant-negative and targeted null mutations in the endothelial receptor tyrosine kinase, tek, reveal a critical role in vasculogenesis of the embryo. Genes Dev. 1994;8(16):1897-909.

27. Jeansson M, et al. Angiopoietin-1 is essential in mouse vasculature during development and in response to injury. J Clin Invest. 2011;121(6):2278-2289.

28. Huang YH, Wu MP, Pan SC, Su WC, Chen YW, Wu LW.STAT1 activation by venous malformations mutant Tie2-R849W antagonizes VEGF-A-mediated angiogenic response partly via reduced $b F G F$ production. Angiogenesis. 2013;16(1):207-222.

29. Morris PN, et al. Functional analysis of a mutant form of the receptor tyrosine kinase Tie2 causing venous malformations. J Mol Med. 2005;83(1):58-63.

30. Uebelhoer M, et al. Venous malformationcausative TIE2 mutations mediate an AKTdependent decrease in PDGFB. Hum Mol Genet. 2013;22(17):3438-3448.

31. Hu HT, Huang YH, Chang YA, Lee CK, Jiang MJ, Wu LW. Tie2-R849W mutant in venous malformations chronically activates a functional STAT1 to modulate gene expression. J Invest Dermatol. 2008;128(9):2325-2333.

32. Korpelainen EI, Karkkainen M, Gunji Y, Vikkula M, Alitalo K. Endothelial receptor tyrosine kinases activate the STAT signaling pathway: mutant Tie- 2 causing venous malformations signals a distinct STAT activation response. Oncogene. 1999;18(1):1-8.

33. Semones M, Feng Y, Johnson N, Adams JL, Winkler J, Hansbury M. Pyridinylimidazole inhibitors of Tie2 kinase. Bioorg Med Chem Lett.
2007;17(17):4756-4760.

34. Greenberger S, Bischoff J. Infantile hemangioma-mechanism(s) of drug action on a vascular tumor. Cold Spring Harb Perspect Med. 2011;1(1):a006460.

35. Phung TL, et al. Pathological angiogenesis is induced by sustained Akt signaling and inhibited by rapamycin. Cancer Cell. 2006;10(2):159-170.

36. Kim I, Kim HG, So JN, Kim JH, Kwak HJ, Koh GY. Angiopoietin-1 regulates endothelial cell survival through the phosphatidylinositol 3'-Kinase/ Akt signal transduction pathway. Circ Res. 2000;86(1):24-29.

37. Kontos CD, et al. Tyrosine 1101 of Tie 2 is the major site of association of $\mathrm{p} 85$ and is required for activation of phosphatidylinositol 3-kinase and Akt. Mol Cell Biol. 1998;18(7):4131-4140.

38. Sarbassov D, et al. Prolonged rapamycin treatment inhibits mTORC2 assembly and Akt/PKB. Mol Cell. 2006;22(2):159-168.

39. Niu XL, Peters KG, Kontos CD. Deletion of the carboxyl terminus of Tie 2 enhances kinase activity, signaling, and function. Evidence for an autoinhibitory mechanism. J Biol Chem 2002;277(35):31768-31773.

40. Shewchuk LM, et al. Structure of the Tie2 RTK domain: self-inhibition by the nucleotide binding loop, activation loop, and C-terminal tail. Structure. 2000;8(11):1105-1113.

41. Heitman J, Movva NR, Hall MN. Targets for cell cycle arrest by the immunosuppressant rapamycin in yeast. Science. 1991;253(5022):905-909.

42. Martel RR, Klicius J, Galet S. Inhibition of the immune response by rapamycin, a new antifungal antibiotic. Can J Physiol Pharmacol. 1977;55(1):48-51

43. Laplante M, Sabatini DM. mTOR signaling in growth control and disease. Cell. 2012;149(2):274-293.

44. Hammill AM, et al. Sirolimus for the treatment of complicated vascular anomalies in children Pediatr Blood Cancer. 2011;57(6):1018-1024.

45. Yuksekkaya H, Ozbek O, Keser M, Toy H. Blue rubber bleb nevus syndrome: successful treatment with sirolimus. Pediatrics. 2012;129(4):4

46. Lackner H, et al. Sirolimus for the treatment of children with various complicated vascular anomalies [published online ahead of print June 4,2015]. Eur JPediatr. doi:10.1007/s00431-015-2572-y.

47. Khan ZA, et al. Multipotential stem cells recapitulate human infantile hemangioma in immunodeficient mice. JClin Invest. 2008;118(7):2592-2599.

48. McHorney CA, Ware JE Jr, Lu JF, Sherbourne CD. The MOS 36-item Short-Form Health Survey (SF-36): III. Tests of data quality, scaling assumptions, and reliability across diverse patient groups. Med Care. 1994;32(1):40-66. 